ARTICLE

\title{
Histone variant H3F3A promotes lung cancer cell migration through intronic regulation
}

Seong-Min Park ${ }^{1, \star}$, Eun-Young Choi ${ }^{1, \star}$, Mingyun Bae ${ }^{2}$, Sunshin Kim ${ }^{3}$, Jong Bae Park ${ }^{1,4}$, Heon Yoo ${ }^{1}$, Jung Kyoon Choi ${ }^{2}$, Youn-Jae Kim ${ }^{1}$, Seung-Hoon Lee ${ }^{1} \&$ In-Hoo Kim ${ }^{4}$

Although several somatic single nucleotide variations in histone $\mathrm{H} 3.3$ have been investigated as cancer drivers, other types of aberration have not been well studied. Here, we demonstrate that overexpression of $\mathrm{H} 3 \mathrm{~F} 3 \mathrm{~A}$, encoding $\mathrm{H} 3.3$, is associated with lung cancer progression and promotes lung cancer cell migration by activating metastasis-related genes. H3.3 globally activates gene expression through the occupation of intronic regions in lung cancer cells. Moreover, $\mathrm{H} 3.3$ binding regions show characteristics of regulatory DNA elements. We show that $\mathrm{H} 3.3$ is deposited at a specific intronic region of GPR87, where it modifies the chromatin status and directly activates GPR87 transcription. The expression levels of H3F3A and GPR87, either alone or in combination, are robust prognostic markers for early-stage lung cancer, and may indicate potential for the development of treatments involving GPR87 antagonists. In summary, our results demonstrate that intronic regulation by H3F3A may be a target for the development of novel therapeutic strategies.

\footnotetext{
${ }^{1}$ Specific Organs Cancer Branch, Research Institute, National Cancer Center, Goyang, Gyeonggi 10408, Republic of Korea. ${ }^{2}$ Department of Bio and Brain Engineering, KAIST, Daejeon 34141, Republic of Korea. ${ }^{3}$ Precision Medicine Branch, Research Institute, National Cancer Center, Goyang, Gyeonggi 10408 , Republic of Korea. ${ }^{4}$ Department of System Cancer Science, Graduate School of Cancer Science and Policy, National Cancer Center, Goyang, Gyeonggi 10408 , Republic of Korea. ${ }^{\star}$ These authors contributed equally to this work. Correspondence and requests for materials should be addressed to Y.-J.K. (email: yjkim@ncc.re.kr) or to S.-H.L. (email: nslsh@ncc.re.kr).
} 
ung cancer is the most frequent cause of cancer-related death in the world, and patients with non-small cell lung cancer (NSCLC) account for $\sim 75 \%$ of lung cancer cases ${ }^{1}$. The risk of relapse after surgery among NSCLC patients is substantial, with $30-35 \%$ of stage I patients relapsing after their initial surgery ${ }^{2,3}$. The subgrouping of early-stage lung cancer patients who will face poor prognoses is important for successful treatment including post-operative adjuvant therapy. Conventionally, a histological subtype marker has been used in post-operative adjuvant therapy, but the effectiveness of this approach is controversial ${ }^{4-7}$. Thus, the identification of novel robust prognostic markers for early-stage lung cancer is imperative.

Histone variants act as transcriptional activators or repressors of cancer-related genes. macroH2A, a non-canonical histone variant, suppresses cancer progression and acts as a direct transcriptional repressor of $C D K 8$, a cancer driver gene ${ }^{8}$. H2A.Z, another histone variant, promotes cancer progression via transcriptional regulation in various cancer types 9,10 . The histone variant $\mathrm{H} 3.3$ is encoded by two genes, $H 3 F 3 A$ and $H 3 F 3 B$. Although only four or five amino acids differ between $\mathrm{H} 3.3$ and the canonical histone $\mathrm{H} 3, \mathrm{H} 3.3$ appears to have different functions in gene regulation ${ }^{11}$. H3.3 is highly enriched in the bodies, promoters and enhancers of actively transcribed genes and is associated with active transcription during various biological processes, including processes associated with development and diseases ${ }^{12-16}$. Recently, highly recurrent somatic missense mutations of $\mathrm{H} 3.3$ genes have been implicated in childhood tumours; in particular, the Lys27Met mutation is a gain-of-function driver mutation that inhibits polycomb repressive complex 2 (PRC2) activity ${ }^{17-20}$. However, the role of differential expression of the H3.3 genes in tumours, particularly adult tumours, has rarely been studied and remains unknown. H3.3 overexpression has been reported in oesophageal and lung cancer cell lines; however, the function of $\mathrm{H} 3.3$ in cancer remains elusive ${ }^{21}$.

Reports have indicated that $\mathrm{H} 3.3$ remodels highly ordered chromatin structures in conjunction with various chromatin remodelers, chaperones and other histone marks, including PRC2, HIRA, CTCF and H2A.Z (refs 15,16,22-25). H3.3 plays different roles in gene transcription depending on its binding position in genome. H3.3 actively marks enhancers and determines the transcriptional potential of target genes; moreover, $\mathrm{H} 3.3$ on promoter regions results in poised or active transcription ${ }^{16}$. Recently, the importance of intronic DNA regulatory elements (IREs) in transcription has been increasingly revealed ${ }^{26,27}$. The transcriptional regulation of IREs is frequently accompanied by histone modification and chromatin remodelling ${ }^{28}$. Certain IREs are associated with cancer progression $^{28}$. Although many researchers have reported the transcriptional regulation activity of $\mathrm{H} 3.3$ on promoter and intergenic enhancer regions, transcriptional regulation via the binding of $\mathrm{H} 3.3$ to the intronic regions of target genes has not been well studied.

In this study, we demonstrate that the overexpression of $\mathrm{H} 3 \mathrm{~F} 3 \mathrm{~A}$ promotes lung cancer cell migration by activating metastasis-related genes. H3.3, which is encoded by $\mathrm{H} 3 \mathrm{~F} 3 \mathrm{~A}$, activates gene expression by occupying intronic regions that exhibit characteristics of regulatory DNA elements. H3.3 directly activates GPR87 transcription via its deposition at a specific intronic region of GPR87, where it modifies chromatin status. H3F3A and GPR87 expression levels, either alone or in combination, are associated with lung cancer patients' prognoses and have potential for the development of treatments involving GPR87 antagonists.

\section{Results}

H3F3A overexpression promotes lung cancer progression. Previous studies addressing the role of H3.3 genes in cancer have primarily focused on recurrent mutations. However, a variety of point mutations and copy number variations (CNV) in $\mathrm{H} 3.3$ genes are found at a low frequency in lung cancer samples, and many well-characterized mutations such as Lys27Met are not recurrent in lung cancer according to publicly available lung cancer data sets from the Catalogue of Somatic Mutations in Cancer database (http://cancer.sanger.ac.uk/cancergenome/ projects/cosmic/, COSMIC; H3F3A: 3/1554 mutations and 15/827 CNV; H3F3B: 4/1553 mutations and 9/827 CNV). Therefore, we examined the association between H3.3 expression and lung cancer progression and evaluated the possibility of using H3.3 genes as prognostic markers for lung cancer. We collected and analysed public microarray lung cancer data sets from the NCBI Gene Expression Omnibus (GEO) database containing clinical information such as cancer stage, recurrence status and survival data. Two data sets from lung adenocarcinomas, GSE13213 and GSE31210, were used in this study because they were already published, and clinical information from a sufficient number of samples was available ${ }^{29,30}$. We performed the analyses using data from all of the patients in each data set (117 patients in GSE13213 and 204 patients in GSE31210) or only data from stage I patients (79 patients in GSE13213 and 162 patients in GSE31210). Comparing $\mathrm{H} 3.3$ gene ( $H 3 F 3 A$ and $H 3 F 3 B)$ expression in non-relapsed and relapsed patients revealed that $H 3 F 3 A$ expression was significantly higher in the relapsed patient group in both data sets (all: $P=1.1 \times 10^{-2}$ for GSE13213 and $P=8.5 \times 10^{-7}$ for GSE31210; stage I: $P=8.5 \times 10^{-3}$ for GSE13213 and $P=2.0 \times 10^{-6}$ for GSE31210, $t$-test), whereas $H 3 F 3 B$ expression was only significantly higher among relapsed patients in the GSE31210 data set (Fig. 1a,b, Supplementary Fig. 1a). Using the survival data, we investigated the association between the survival rate for lung adenocarcinoma patients after surgery and H3.3 gene expression (Fig. 1c,d, Supplementary Fig. 1b). Survival was significantly lower in the group with higher $H 3 F 3 A$ expression than it was in the lower expression group in both data sets (all: $P=3.2 \times 10^{-2}$ for GSE13213, $P=9.7 \times 10^{-3}$ for GSE31210 overall survival, $P=6.4 \times 10^{-2}$ for GSE31210 relapse-free survival; stage I: $P=2.7 \times 10^{-2}$ for GSE13213, $P=1.2 \times 10^{-2}$ for GSE31210 overall survival, $P=6.3 \times 10^{-4}$ for GSE31210 relapse-free survival, log-rank test), whereas the higher $H 3 F 3 B$ expression group exhibited significantly lower survival only in the GSE31210 data set. The expression of canonical histone $\mathrm{H} 3$ genes was not significantly associated with relapse or survival (Supplementary Figs 2 and 3). Based on these results, we suggest that $H 3 F 3 A$ overexpression in lung cancer promotes cancer progression, and we subsequently focused on $\mathrm{H} 3 \mathrm{~F} 3 \mathrm{~A}$ and stage I lung cancer.

To assess the value of $H 3 F 3 A$ expression as a prognostic marker for stage I lung adenocarcinoma, we performed a multivariate Cox proportional hazard analysis using the two public data sets (Table 1). The level of H3F3A expression better predicted poor survival (low survival rate) in both data sets than other markers, including histological grouping into stage IA and stage IB. Thus, we suggest that $H 3 F 3 A$ overexpression is a good prognostic marker for early-stage lung cancer.

H3F3A overexpression promotes cancer cell invasion. Additional clinical information about relapse sites was available in the GSE13213 data set, which revealed that all but six relapsed patients exhibited distant metastases. As $H 3 F 3 A$ overexpression was associated with relapse and poor prognosis (low survival rate) in lung cancer patients, we ectopically overexpressed $\mathrm{H} 3 \mathrm{~F} 3 \mathrm{~A}$ in 


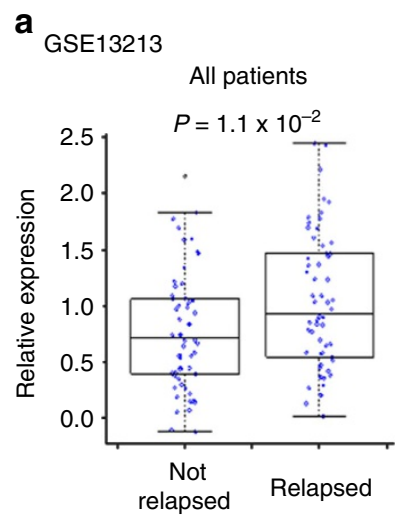

C
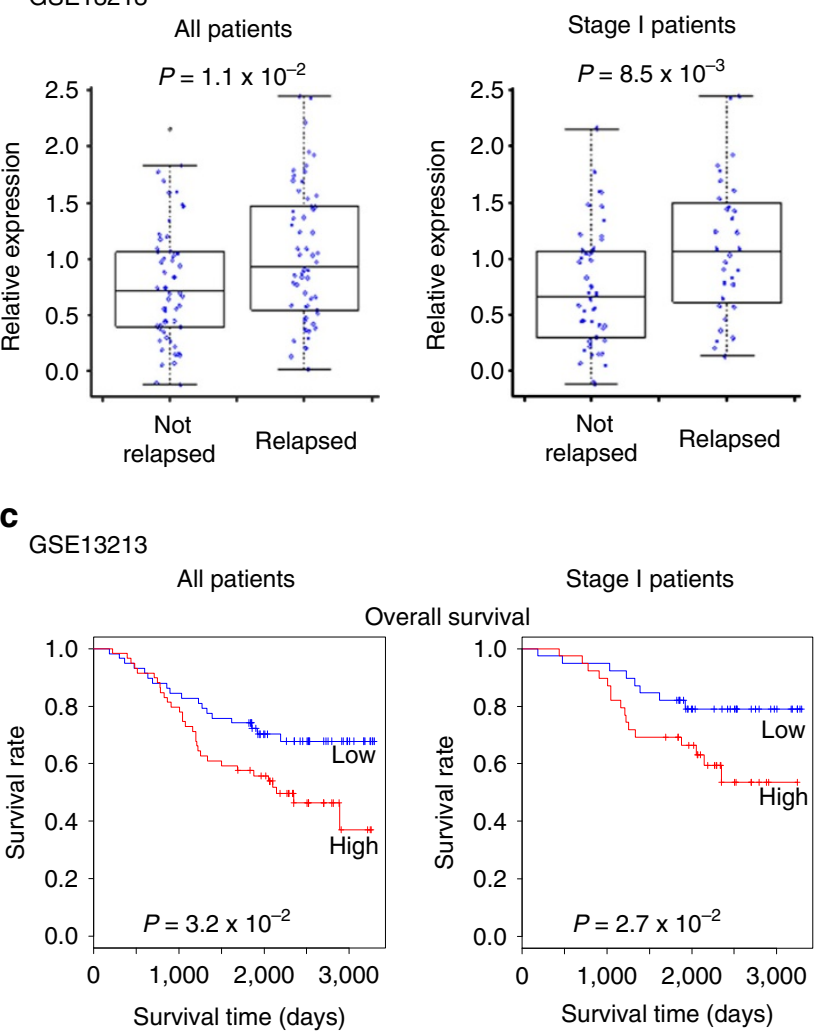

b
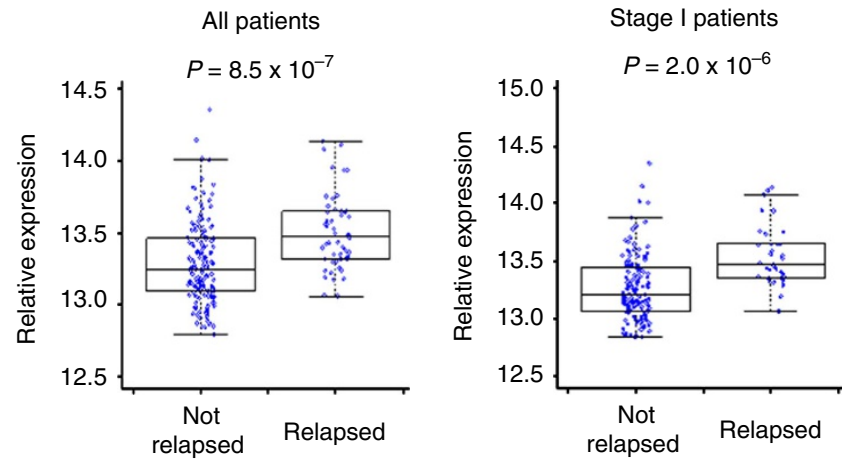

d

GSE31210

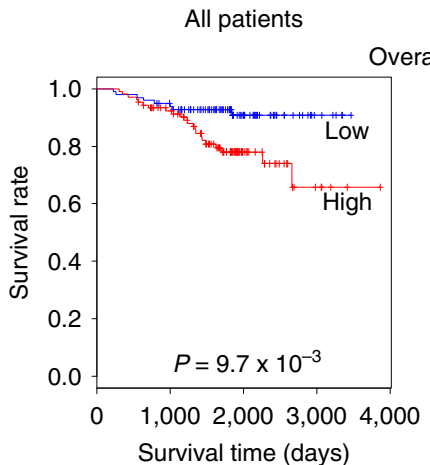

Stage I patients

Overall survival

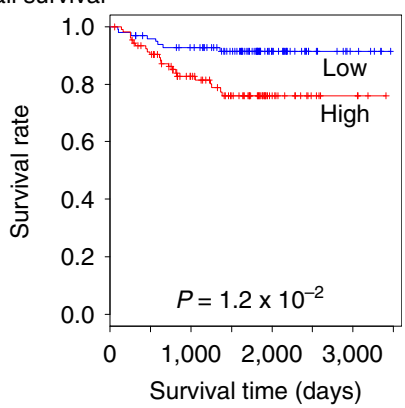

Relapse-free survival

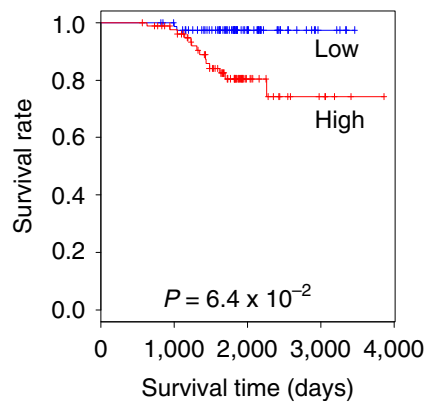

Figure 1 | Promotion of lung cancer progression by H3F3A overexpression. (a) Comparison of H3F3A expression in relapsed and non-relapsed lung cancer patients (GSE13213, Tomida et al. ${ }^{29}$. All: $n=117, P=1.1 \times 10^{-2}$, stage I: $n=79, P=8.5 \times 10^{-3}, t$-test). (b) (GSE31210, Okayama et al. ${ }^{30}$. All: $n=204, P=8.5 \times 10^{-7}$, stage $\mathrm{I}: n=162, P=2.0 \times 10^{-6}, t$-test). (c) Prognosis of two groups of lung cancer patients classified by H3F3A expression (GSE13213, Tomida et al. ${ }^{29}$. All: $n=117, P=3.2 \times 10^{-2}$, stage I: $n=79, P=2.7 \times 10^{-2}$, log-rank test). (d) (GSE31210, Okayama et al. ${ }^{30}$. All: $n=204$, $P=9.7 \times 10^{-3}$ for overall survival, $P=6.4 \times 10^{-2}$ for relapse-free survival; stage $I: n=162, P=1.2 \times 10^{-2}$ for overall survival, $P=6.3 \times 10^{-4}$ for relapse-free survival, log-rank test). Red: high expression group, blue: low expression group.

human lung cancer cell lines and examined several cancer-related phenotypes. To evaluate metastasis-related phenotypes, we performed an invasion assay using $H 3 F 3 A$-overexpressing A549 and NCI-H23 cells (Fig. 2a,c). H3F3A overexpression significantly increased lung cancer cell invasion $\left(P=2.0 \times 10^{-5}\right.$ for A549 and $P=7.7 \times 10^{-6}$ for NCI-H23, $t$-test). We also performed an invasion assay using H3F3A-knockdown A549 and NCI-H23 cells (Fig. 2b,d). As expected, H3F3A knockdown significantly decreased invasion $\left(P=1.0 \times 10^{-5}\right.$ for A549 and $P=1.7 \times 10^{-8}$ for NCI-H23, $t$-test). Furthermore, we performed invasion assays using canonical histone H3-overexpressing A549 cells (Supplementary Fig. 4). Overexpression of the H3.1 and H3.2 genes did not have significant effects on the invasion of A549 cells. Next, we investigated changes in the expression of MMP9, a well-known invasion-related effector gene, following $\mathrm{H} 3 \mathrm{~F} 3 \mathrm{~A}$ overexpression or knockdown (Fig. 2). As expected,
MMP9 expression was increased by $H 3 F 3 A$ overexpression (overexpression: $P=1.7 \times 10^{-3}$ for A549 and $P=9.7 \times 10^{-5}$ for NCI-H23, $t$-test; knockdown: $P=3.8 \times 10^{-2}$ for A549 and $P=1.3 \times 10^{-3}$ for NCI-H23, $t$-test). We next examined growthrelated phenotypes by performing cell proliferation assays, although we did not observe any significant changes in proliferation following $H 3 F 3 A$ overexpression or knockdown (Fig. 2, bottom). Based on these results, we concluded that H3F3A overexpression in lung cancer cells promotes cancer cell invasion, which is important for cancer metastasis and progression.

Transcriptional regulation by $\mathbf{H} 3 \mathbf{F} 3 \boldsymbol{A}$ in lung cancer. $H 3 F 3 A$ encodes the histone variant $\mathrm{H} 3.3$, the general function of which is to regulate transcription. Thus, we hypothesized that H3.3 functions in lung cancer by transcriptionally regulating downstream target genes. To identify $H 3 F 3 A$ target genes, we 


\begin{tabular}{|c|c|c|}
\hline \multirow[t]{2}{*}{ Variable } & \multicolumn{2}{|c|}{ Survival } \\
\hline & HR $(95 \% \mathrm{Cl})$ & $P$ value \\
\hline \multicolumn{3}{|l|}{ GSE13213 overall } \\
\hline H3F3A & $2.80(1.11-7.03)$ & 0.0288 \\
\hline p53 mutation & $1.71(0.251-1.36)$ & 0.212 \\
\hline Stage IA versus IB & $1.68(0.718-3.94)$ & 0.231 \\
\hline EGFR mutation & $1.63(0.604-4.40)$ & 0.334 \\
\hline Smoking & $1.00(0.999-1.00)$ & 0.504 \\
\hline H3F3B & $1.25(0.340-1.89)$ & 0.614 \\
\hline K-ras mutation & $1.29(0.228-2.66)$ & 0.689 \\
\hline \multicolumn{3}{|l|}{ GSE31210 overall } \\
\hline H3F3A & $5.71(1.24-26.3)$ & 0.0256 \\
\hline H3F3B & $3.80(1.04-13.9)$ & 0.0434 \\
\hline EGFR mutation & $2.03(0.174-1.39)$ & 0.182 \\
\hline Stage IA versus IB & $1.52(0.53856-4.267)$ & 0.431 \\
\hline MYC expression & $1.46(0.294-7.26)$ & 0.644 \\
\hline MYC copy & $1.24(0.399-3.84)$ & 0.711 \\
\hline K-ras mutation & $1.49(0.0783-5.77)$ & 0.717 \\
\hline Smoking & $1.18(0.287-2.50)$ & 0.765 \\
\hline ALK fusion & $1.47 \times 10-7(0-\infty)$ & 1.00 \\
\hline \multicolumn{3}{|l|}{ GSE31210 relapse-free } \\
\hline H3F3A & $6.68(1.46-30.4)$ & 0.0141 \\
\hline H3F3B & $3.24(0.885-11.9)$ & 0.0758 \\
\hline EGFR mutation & $2.10(0.169-1.34)$ & 0.159 \\
\hline Stage IA versus IB & $1.90(0.653-5.51)$ & 0.240 \\
\hline K-ras mutation & $1.52(0.0774-5.60)$ & 0.702 \\
\hline MYC copy & $1.06(0.337-3.36)$ & 0.916 \\
\hline Smoking & $1.05(0.322-2.82)$ & 0.930 \\
\hline MYC expression & $1.07(0.183-4.77)$ & 0.935 \\
\hline ALK fusion & $4.91 \times 10-7(0-\infty)$ & 1.00 \\
\hline
\end{tabular}

performed microarray-based global gene expression analysis of H3F3A-overexpressing and H3F3A knockdown A549 cells. Genes that exhibited $>2$-fold changes in expression following either overexpression or knockdown, with opposite responses in the overexpression and knockdown treatments, were identified as differentially expressed genes (DEGs) (Fig. 3a,b). A total of 112 positively regulated DEGs (Supplementary Data 1) and 70 negatively regulated DEGs were selected (Supplementary Data 2). The expression patterns of some candidate genes were validated by quantitative reverse transcription PCR (RT-qPCR; Supplementary Fig. 5) and semi-quantitative RT-PCR (RT-PCR; Fig. 3c). We performed Gene Ontology (GO) analysis of the selected DEGs (Fig. 3d and Supplementary Data 3 and 4), and the top-ranked Gene Ontology term among the genes that were positively regulated by $H 3 F 3 A$ expression was 'collagen metabolic process,' which included several well-known metastasis-related effector genes, such as metallomatrix proteases. We did not observe any metastasis-related terms for the negatively regulated genes, leading us to focus on the positively regulated genes in the subsequent analysis. Following further analysis using the Gene Set Enrichment Analysis programme, we also found that genes that were positively regulated by $H 3 F 3 A$ were significantly associated with the 'Epithelial Mesenchymal Transition' and 'Metallopetidase Activity' gene sets, which are closely involved in cancer metastasis (Fig. 3e). Therefore, we concluded that genes that are positively regulated by $H 3 F 3 A$ are closely associated with metastasis-related processes.
To identify genes that are occupied by $\mathrm{H} 3.3$ following $\mathrm{H} 3 \mathrm{~F} 3 \mathrm{~A}$ overexpression, we performed chromatin immunoprecipitation (ChIP)-seq using H3F3A-overexpressing A549 lung cancer cells. In total, $871 \mathrm{H} 3.3$ peaks were detected following overexpression using a high stringency cutoff, and we then annotated these peaks by genomic position (Fig. 4a). The majority of the peaks localized to intergenic and intronic regions (intergenic: $\sim 56 \%$, intronic: $\sim 39 \%$ ). To examine the correlation between $\mathrm{H} 3.3$ occupancy and target gene expression, we identified the top 3,000 peaks that were located within introns, promoters and intergenic regions using a low-stringency cutoff. The target genes of intergenic peaks could not be identified without additional data. Thus, we defined the nearest genes within $10 \mathrm{~kb}$ from the peaks as the target genes, assuming that the peaks corresponded to cis-regulatory elements. We examined the H3.3 ChIP-seq read distribution near each H3.3 summit, and after calculating a normalized read count around each of the 3,000 H3.3 summits located within introns, promoters and intergenic regions (from $-5 \mathrm{~kb}$ to $+5 \mathrm{~kb}$ ), we sorted the peaks based on target gene expression level (Fig. 4b). Additionally, after separating the peaks into three groups based on the expression of the target genes (high, intermediate and low expression groups), we calculated average normalized read counts for these groups near the H3.3 summits (from $-2 \mathrm{~kb}$ to $+2 \mathrm{~kb}$; Fig. 4c). In both analyses, H3.3 intronic peaks were positively correlated with target gene expression, whereas promoter and intergenic peaks showed no such correlation. Thus, it appears that H3.3 occupancy of introns activates the expression of target genes.

To perform a further analysis, we downloaded histone ChIP-seq data from the Encyclopedia of DNA Elements (ENCODE) database (https://genome.ucsc.edu/ENCODE/). Because H3.3 binding at introns was correlated with target gene expression, we examined with which histone marks $\mathrm{H} 3.3$ was associated (Fig. 4d). In A549 lung cancer cells, the top 3,000 intronic $\mathrm{H} 3.3$ peaks most frequently overlapped with peaks of mono-methylation of histone $\mathrm{H} 4$ lysine 20 (H4K20me1), tri-methylation of histone $\mathrm{H} 3$ lysine 36 (H3K36me3) and di-methylation of histone $\mathrm{H} 3$ lysine 79 (H3K79me2), as these marks are gene body histone marks. Aside from the gene body marks, intronic H3.3 most frequently bound to the enhancer-specific histone mark mono-methylated histone H3 lysine 4 (H3K4me1), suggesting that intronic $\mathrm{H} 3.3$ tends to bind enhancers.

Transcriptional regulation of GPR87 by H3F3A. To select direct transcriptional targets of $H 3 F 3 A$, we performed a Venn analysis using the ChIP-seq and microarray data. Considering that intronic $\mathrm{H} 3.3$ frequently bound to enhancer-specific histone mark H3K4me1, as shown in Fig. 4d, we selected 787 genes occupied by both $\mathrm{H} 3.3$ and $\mathrm{H} 3 \mathrm{~K} 4 \mathrm{mel}$ peaks from the 2,392 genes that are occupied by the top 3,000 intronic H3.3 peaks. Performing Venn analysis with the 787 genes from ChIP-seq and the DEGs using low-stringency cutoffs (top 3,000 probes) from the microarray, we found 29 putative target genes exhibiting H3.3-occupied intronic regulation (Supplementary Fig. 6). Using high stringency cutoffs, we performed another Venn analysis. Performing Venn analysis with the 366 genes occupied by the high stringency $\mathrm{H} 3.3$ peaks and the DEGs showing $>2$-fold changes in expression following H3F3A overexpression or knockdown, we selected one putative target gene, G-protein-coupled receptor 87 (GPR87) (Fig. 5a). GPR87 was also included in the 29 low-stringency putative target genes. The differential expression of GPR87 by H3F3A overexpression and knockdown was validated using RT-qPCR (Fig. 5b). Moreover, we confirmed specific H3.3 binding to the GPR87 intron using a ChIP-PCR assay (Fig. 5c). As GPR87 is 
a

A549

Immunoblot
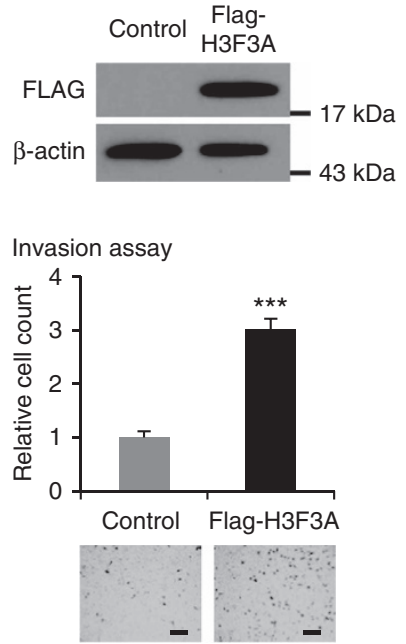

RT-qPCR (MMP9)

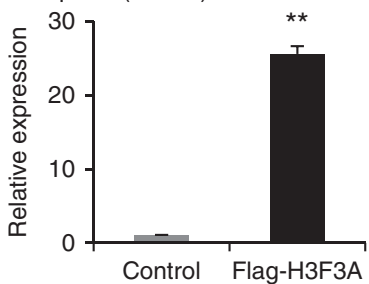

Proliferation assay

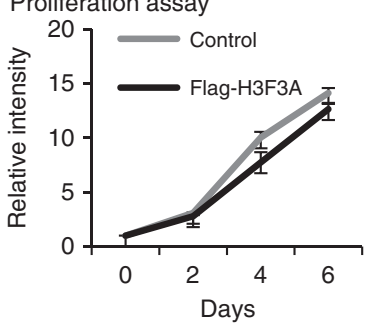

b

RT-PCR
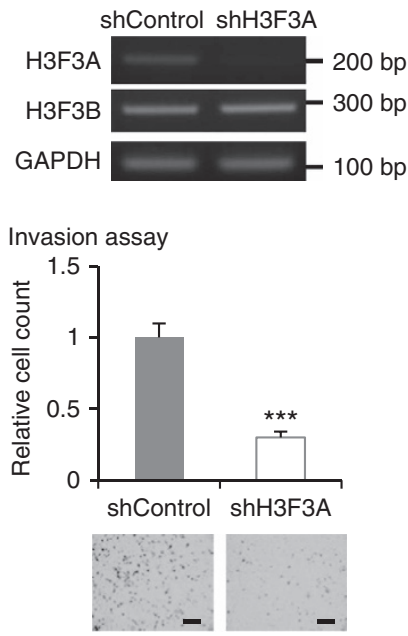

RT-qPCR (MMP9)
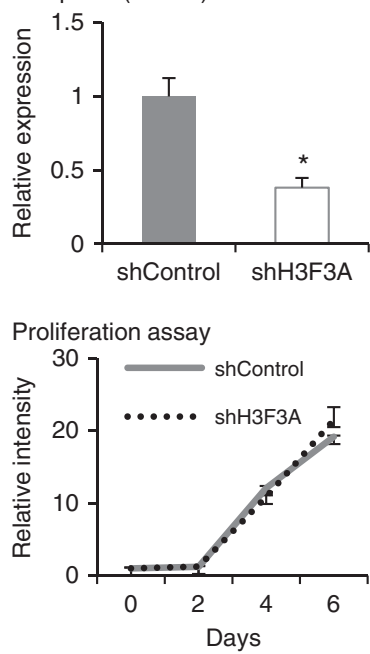

C

$\mathrm{NCl}-\mathrm{H} 23$

Immunoblot Control Flag-
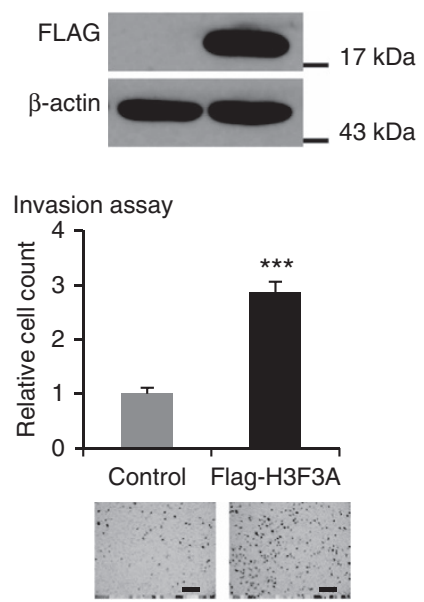

RT-qPCR (MMP9)

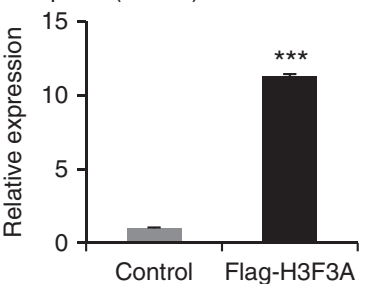

Proliferation assay

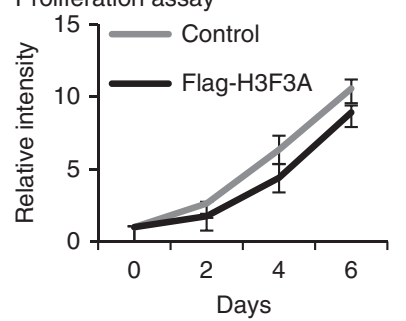

d

RT-PCR
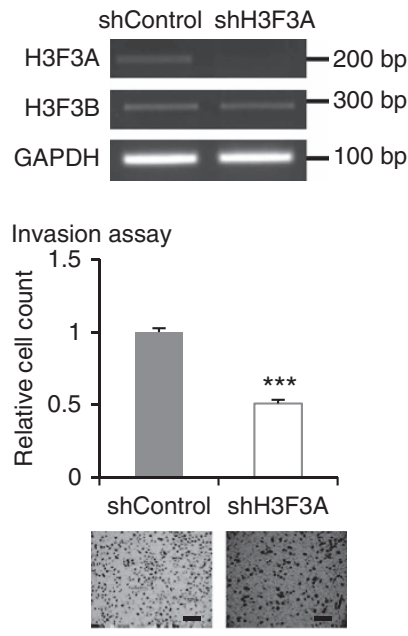

RT-qPCR (MMP9)
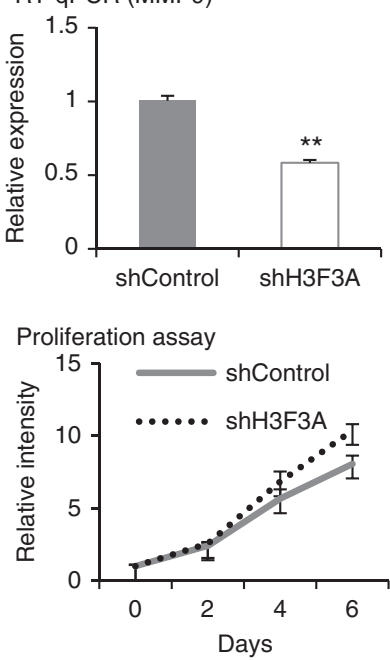

Figure 2 | Promotion of lung cancer cell invasion by H3F3A overexpression. (a) Results of invasion assay, MMP9 RT-qPCR and proliferation assay after ectopic H3F3A overexpression in A549 cells. (b) After $\mathrm{H} 3 \mathrm{~F} 3 \mathrm{~A}$ knockdown in A549 cells. (c) After ectopic $\mathrm{H} 3 \mathrm{~F} 3 \mathrm{~A}$ overexpression in $\mathrm{NCl}-\mathrm{H} 23$ cells. (d) After H3F3A knockdown in NCl-H23 cells. Data are representative of three independent experiments. The error bars represent the s.e.m. ${ }^{\star} P<0.05$, ${ }^{\star \star} P<0.01,{ }^{\star \star \star} P<0.001, t$-test. Scale bar, $200 \mu \mathrm{m}$.

known to be associated with cancer progression in NSCLC (ref. 31), we focused on GPR87 in the subsequent analysis.

As we expected that $H 3 F 3 A$ would promote lung cancer cell invasion through GPR87, we examined whether the increased invasion ability stimulated by $H 3 F 3 A$ overexpression could be mitigated by GPR87 knockdown (Fig. 5d). GPR87 knockdown strongly suppressed the increased invasion ability induced by H3F3A overexpression $\left(P=2.0 \times 10^{-3}, t\right.$-test $)$. In addition, double H3F3A/GPR87 knockdown did not further potentiate the effect on the invasion of single knockdowns (Supplementary Fig. 7). We concluded that increased GPR87 expression caused by $H 3 F 3 A$ overexpression promotes the invasion ability of lung cancer cells.

Occupancy of $\mathrm{H} 3.3$ at the GPR87 intronic region has been observed in various public data sets, such as NCBI GEO GSE31794 and GSE45023 data sets for HeLa cervical cancer cells $^{23,32}$ (Fig. 5e). H3.3 ChIP-seq data sets for lung cancer cells were not available, prompting us to use H3.3 ChIP-seq data sets from HeLa cervical cancer cells. The integrated analysis suggested that the H3.3-occupied GPR87 intronic region is an intronic regulatory DNA element (IRE), as it is located in an open chromatin region that is bound to more transcription factors than the GPR87 promoter region, and is also occupied by active histone marks such as $\mathrm{H} 2 \mathrm{~A}$ histone family member $\mathrm{Z}$ (H2A.Z), mono- and tri-methylation of histone $\mathrm{H} 3$ lysine 4 (H3K4me1 and H3K4me3) and acetylation of histone H3 lysine 9 and lysine 27 (H3K9ac and H3K27ac) (Fig. 5e). Tri-methylation of histone H3 lysine 27 (H3K27me3), a repressive histone mark, was not observed in this region. To examine changes in chromatin status at the GPR87 IRE, we performed a ChIP-qPCR assay to analyse changes in several histone marks (Fig. 5f). H3F3A overexpression increased the occupancy of three types of histone marks: H2A.Z (an active histone variant), H3K4me1 (an enhancer-specific histone mark) and $\mathrm{H} 3 \mathrm{~K} 9 \mathrm{ac}$ (a histone mark of active promoters and enhancers) ${ }^{33}$. These results indicate that H3F3A overexpression modifies the chromatin status near the GPR87 IRE, demonstrating that this is a true H3.3-occupied IRE.

To perform a further integrated analysis, we downloaded another public H3.3 ChIP-seq data set from the NCBI sequence read archive (SRA) database: SRA043915 (ref. 34). The study of 
a

DEG selection
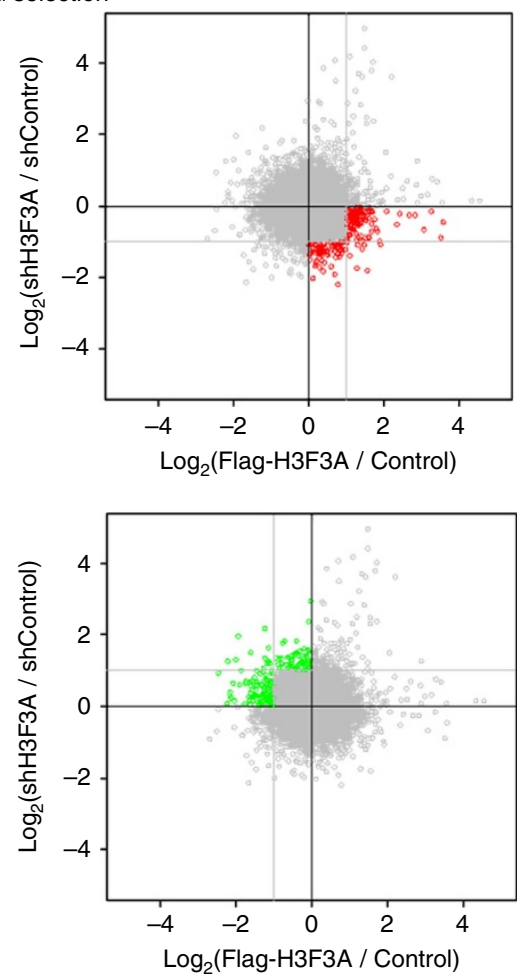

b

Clustering

RT-PCR
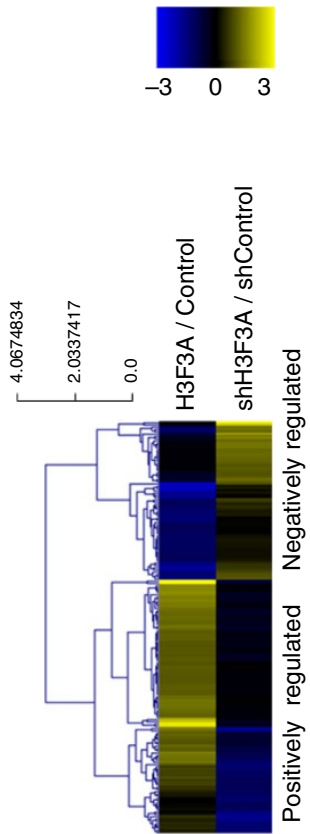

d

GO Biological process (Microarray)

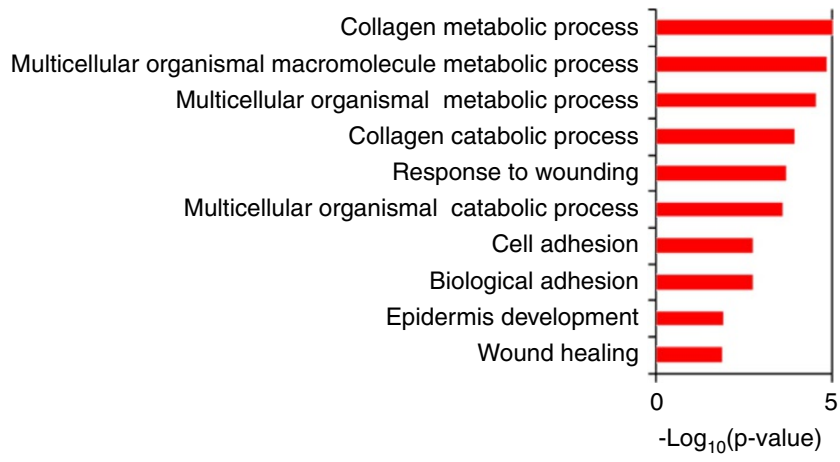

Protein polymerization

Platelet activation

Regulation of body fluid levels

Regulation of smooth muscle cell proliferation

Cellular protein complex assembly

Response to calcium ion

Response to wounding

Response to inorganic substance

Blood coagulation

Coagulation

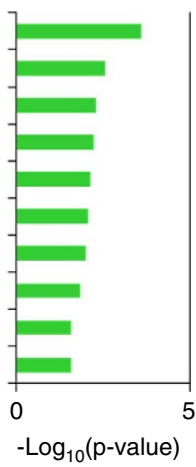

e

GSEA

Hallmark:

epithelial mesenchimal transition

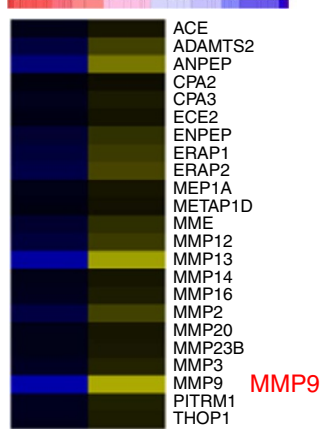

Control H3F3A

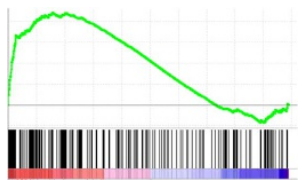

GO molecular function:

Metallopetidase activity
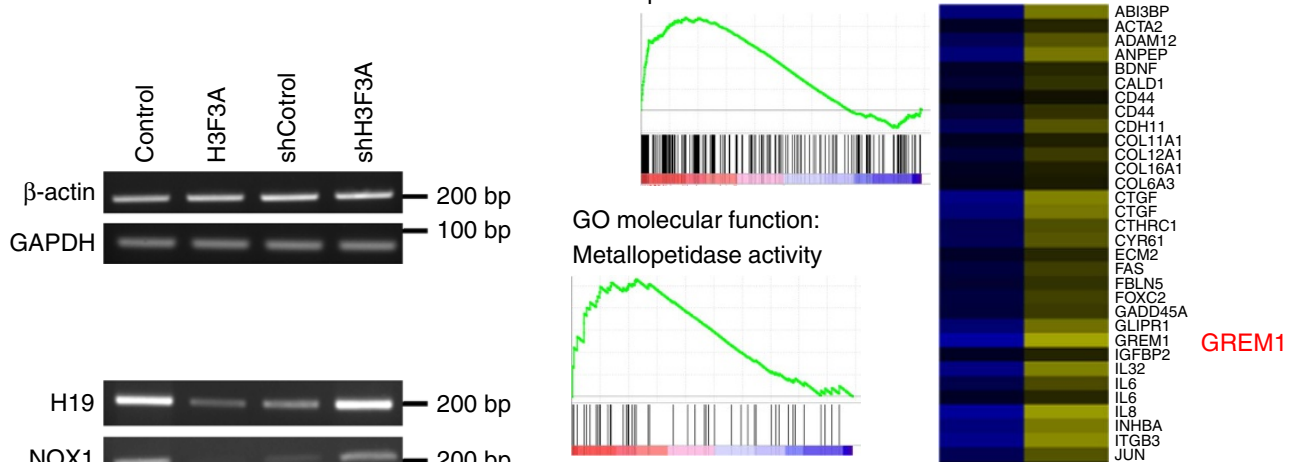

GREM1

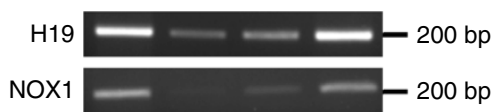

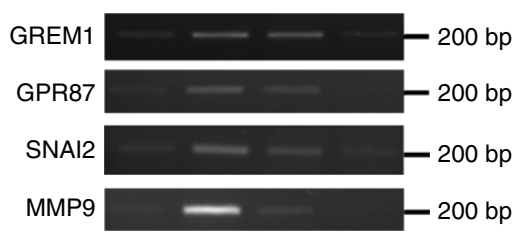

Figure 3 | Microarray-based global analysis for transcriptional regulation via H3F3A. (a) Selection of DEGs using the expression microarray analysis in H3F3A-altered A549 cells. (b) Expression pattern of the DEGs. (c) Validation of DEGs using RT-PCR. (d) GO analysis result of the DEGs (GO_BP).

(e) Gene set enrichment analysis result of the positively correlated DEGs. 
a

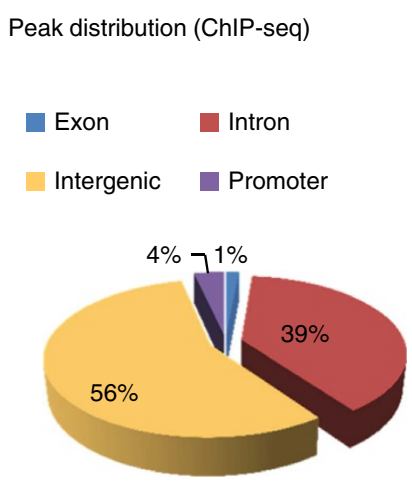

b

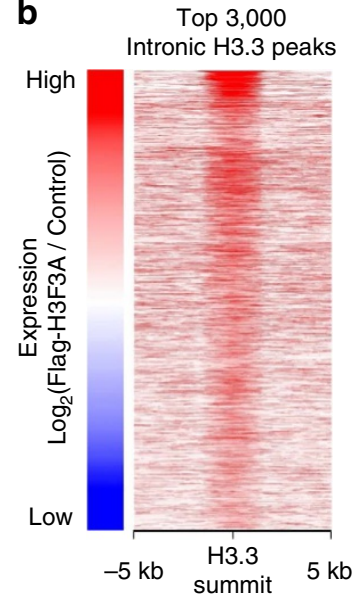

Top 3,000

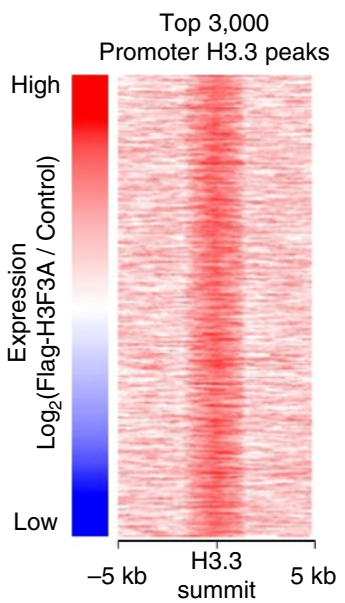

Top 3,000

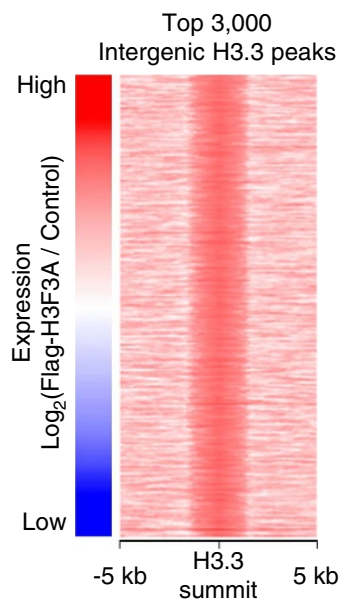

C

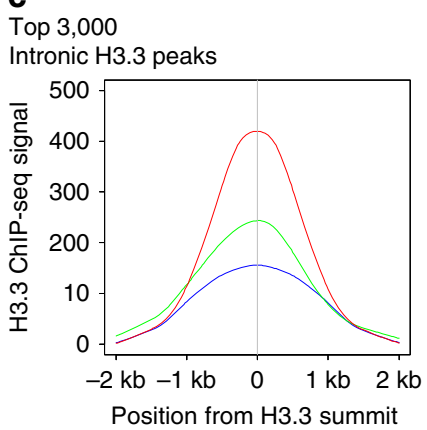

Top 3,000

Promoter $\mathrm{H} 3.3$ peaks

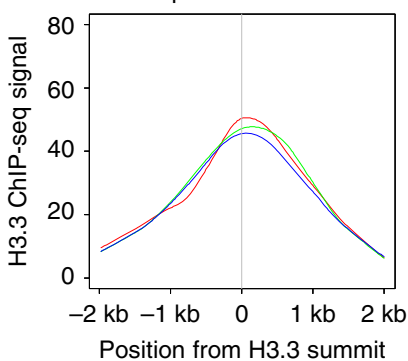

Expression level:
Top 3,000

Intergenic $\mathrm{H} 3.3$ peaks

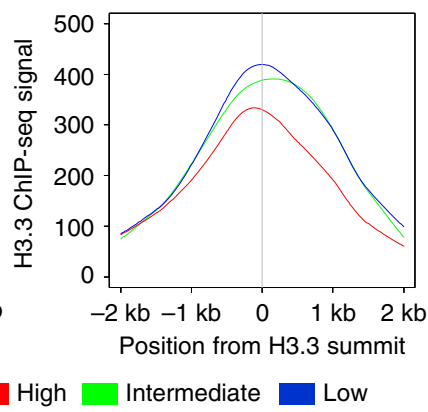

d

Top 3,000

Intronic $\mathrm{H} 3.3$ peaks

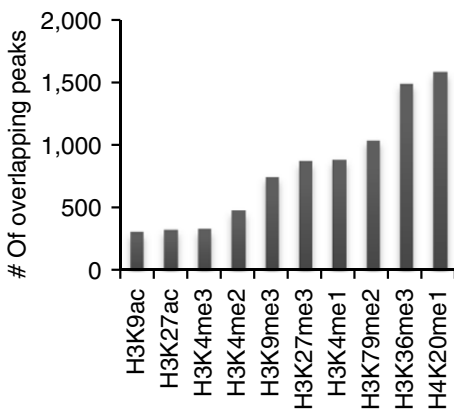

Figure 4 | Chip-seq-based global analysis for transcriptional regulation via H3F3A. (a) Genomic distribution of H3.3 ChIP-seq peaks in H3F3A-overexpressed A549 cells. (b) Correlation between H3.3 occupancy and target gene expression at intron, promoter and intergenic region. (c) H3.3 binding profile of intronic/promoter/intergenic peaks of three gene groups classified by the expression level (red: high expression group, yellow-green: intermediate expression group, blue: low expression group). (d) Association of H3.3 peaks with various histone marks.

SRA043915 allowed us to separate high H3.3 nucleosome turnover regions from the rest of the genome. Interestingly, GPR87 IRE overlapped with a high H3.3 nucleosome turnover region (Fig. 5e), which was reported to be associated with active histone modification marks at promoters and enhancers ${ }^{35}$, consistent with our result. Then, we performed another global analysis using the SRA043915 data, our own H3.3 ChIP-seq data for A549 cells and the ENCODE ChIP-seq data for A549 cells. After calculating normalized read counts for histone marks near each of the 871 high-stringency $\mathrm{H} 3.3$ summits (from $-1 \mathrm{~kb}$ to $+1 \mathrm{~kb}$ ), we compared the occupancy of histone marks on H3.3 peaks that overlapped with high turnover regions with that for low turnover regions (Supplementary Fig. 8). The H3.3 peaks that overlapped with high turnover regions showed higher occupancy of active histone marks, such as H2A.Z, H3K4me1, H3K4me3, $\mathrm{H} 3 \mathrm{~K} 9 \mathrm{ac}$ and $\mathrm{H} 3 \mathrm{~K} 27 \mathrm{ac}$, and lower occupancy for repressive histone marks, such as tri-methylation of histone $\mathrm{H} 3$ lysine 9 (H3K9me3) and $\mathrm{H} 3 \mathrm{~K} 27 \mathrm{me} 3$, compared with the $\mathrm{H} 3.3$ peaks that overlapped with low turnover regions. The high turnover H3.3 peaks show characteristics of active regulatory DNA elements. These results are also consistent with the previous report ${ }^{35}$.

The previous results indicated that the GPR87 IRE could be an intronic hotspot for transcriptional regulation. To examine the functional role of the GPR87 IRE, we constructed reporters containing the GPR87 IRE (154 bp), a non-IRE sequence (152 bp, located at $1,703 \mathrm{bp}$ upstream from the GPR87 IRE) and no sequence (Fig. 6a,c). The GPR87 and SV40 promoters were inserted into the constructs to examine promoter dependence.
Luciferase assays using these reporters revealed that the GPR87 IRE suppressed reporter gene expression, whereas the non-IRE sequence had no effect (Fig. 6b,d). Interestingly, $H 3 F 3 A$ overexpression at least partially rescued this suppression of gene expression (Fig. 6b,d). The effects of both GPR87 IRE and H3F3A overexpression were independent of promoter type. Therefore, we concluded that H3F3A overexpression increases GPR87 expression by suppressing the inhibitory activity of the GPR87 IRE.

To examine chromatin interactions between the GPR87 IRE and transcription start site (TSS), we performed a chromatin conformation capture (3C) PCR assay with primers specific to the GPR87 IRE, non-IRE and TSS (Fig. 6e). H3F3A overexpression increased chromatin interactions between the GPR87 IRE and TSS, suggesting that H3.3 binding to the GPR87 IRE generates a physical interaction between the GPR87 IRE and TSS. Next, we evaluated whether changes at the GPR87 IRE caused by $\mathrm{H} 3 \mathrm{~F} 3 \mathrm{~A}$ overexpression influence the transcriptional machinery at the GPR87 TSS. By performing a ChIP-qPCR assay near the TSS, we found that $H 3 F 3 A$ overexpression increased the occupancy of RNA polymerase II (Pol II) and phosphorylated RNA polymerase II (Pol II phospho S5) at the proximal $(-113$ to $+36 \mathrm{bp}$ around the TSS) and distal ( -898 to -733 bp upstream of the TSS) GPR87 promoters (Fig. 6f). These results demonstrate that H3.3 binding to the GPR87 IRE directly regulates the transcriptional machinery at GPR87.

HIRA and DAXX/ATRX are known as the histone chaperone complex, which recruits assembly factors for H3.3 deposition ${ }^{15,24}$. 
a
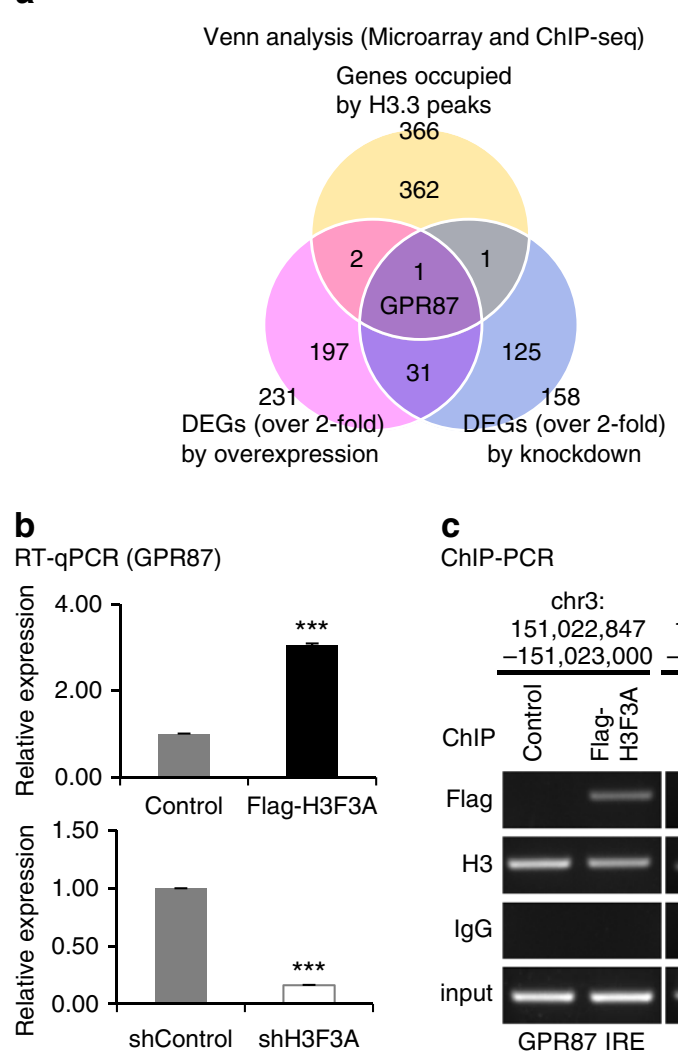

C

ChIP-PCR

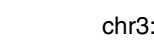

chr3: chr3:

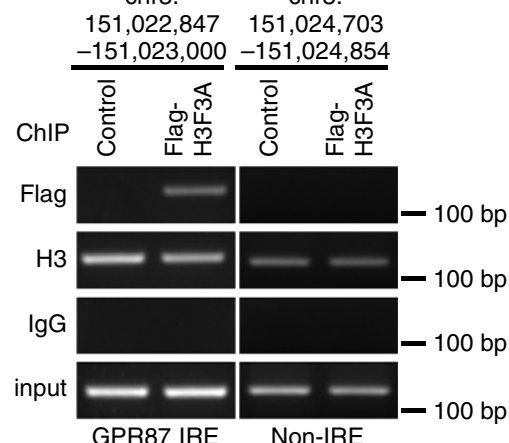

d

Invasion Assay

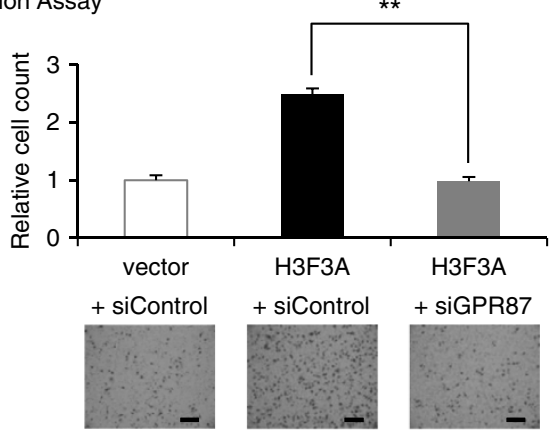

\section{f} ChIP qPCR e

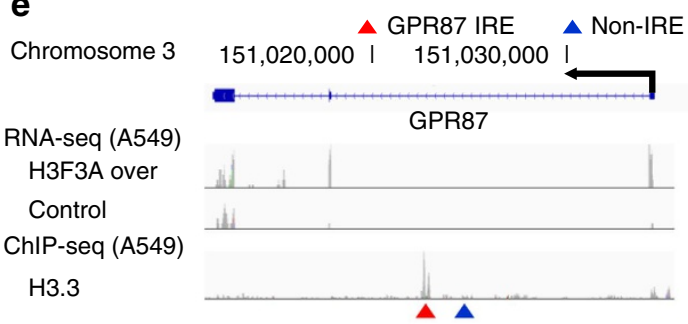

ChIP-seq (GSE31794, Ray-Gallet et al. 2011)

H3.3

ChIP-seq (GSE45023, Pchelintsev et al. 2013)

H3.3

ENCODE Open Chromatin (A549)

DNase-seq

ENCODE ChIP-seq (A549)

H2A.Z

H3K4me1

$\mathrm{H} 3 \mathrm{~K} 4 \mathrm{me} 3$

H3K9ac

H3K27ac

H3K27me3

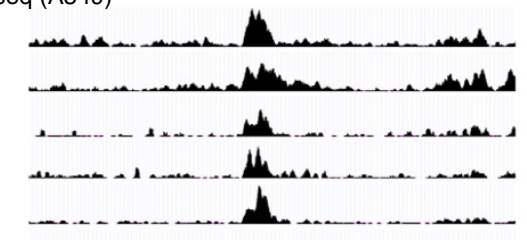

ENCODE

TF binding

(A549 cell)
ChIP-seq(SRA043915, Huang et al. 2013) High H3.3 turnover

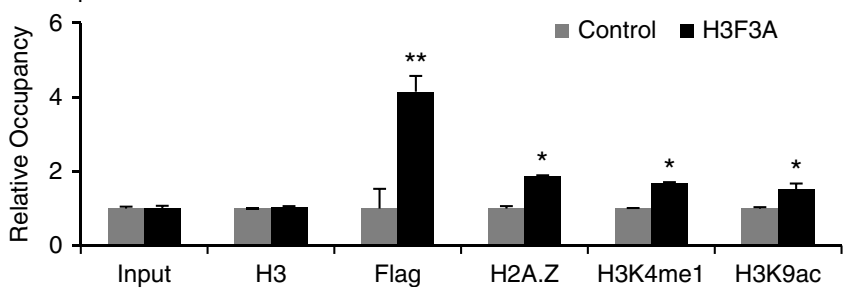

Figure 5 | Selection and validation of an H3.3 target gene. (a) Selection of putative direct target genes of H3F3A with microarray and ChIP-seq data. (b) RT-qPCR validation of the changes in the expression of GPR87 induced by H3F3A overexpression and knockdown. (c) Validation of H3.3 binding to the IRE of GPR87 using a ChIP-PCR assay (GPR87 IRE: chr3:151,022,847-151,023,000; Non-IRE: chr3:151,024,703-151,024,854). (d) Results of invasion assays after ectopic H3F3A overexpression and GPR87 knockdown. (e) Integrated analysis near GPR87 gene. (f) Chromatin status modification at GPR87 IRE due to ectopic H3F3A overexpression. Data are representative of three independent experiments. The error bars represent the s.e.m. ${ }^{\star} P<0.05$, ${ }^{\star \star} P<0.01$,

${ }_{\star \star *} P<0.001, t$-test. Scale bar, $200 \mu \mathrm{m}$.

To determine which $\mathrm{H} 3.3$ chaperone is involved in regulating invasion ability, we performed invasion assays with $H 3 F 3 A$ overexpressing cells after DAXX and HIRA short interfering RNA (siRNA) treatment (Fig. 6g). Knockdown of DAXX did not influence invasion ability, but HIRA knockdown significantly decreased the enhanced invasion caused by H3.3 overexpression. To examine whether HIRA is involved in promoting H3.3 loading at the GPR87 IRE, we performed a ChIP-qPCR assay with $H 3 F 3 A$ overexpression after HIRA knockdown (Fig. 6h). Knockdown of HIRA significantly decreased H3.3 binding at the GPR87 IRE. These results imply that HIRA acts as a chaperone for $\mathrm{H} 3.3$ loading at the GPR87 IRE. The expression levels of DAXX and HIRA were not significantly associated with the survival of lung cancer patients (Supplementary Fig. 9).
Lung cancer promotion by the combination of H3F3A and GPR87. Based on previous results, the transcriptional regulation of GPR87 by H3F3A appears to be essential for the invasion ability of lung cancer cells. Thus, we attempted to characterize the clinical relevance of GPR87 transcriptional regulation by $H 3 F 3 A$ overexpression. An analysis of the data sets shown in Fig. 1 revealed that GPR87 expression is significantly associated with both relapse and prognosis in stage I lung adenocarcinoma patients (Fig. 7a,b; relapse: $P=4.0 \times 10^{-3}$ for GSE13213 and $P=7.8 \times 10^{-4}$ for GSE31210, $t$-test; survival: $P=9.0 \times 10^{-3}$ for GSE13213, $3.1 \times 10^{-2}$ for overall survival of GSE31210 and $P=2.9 \times 10^{-3}$ for relapse-free survival of GSE31210, log-rank test). Therefore, we suggest that GPR87 expression changes driven by $H 3 F 3 A$ overexpression promote lung cancer progression. 
a

Reporter construct

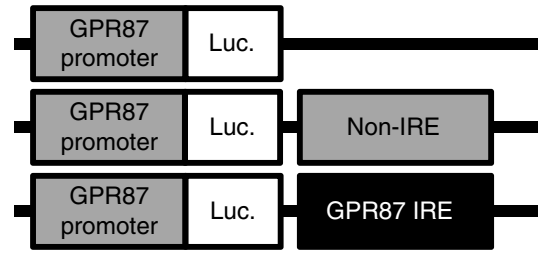

C

Reporter construct

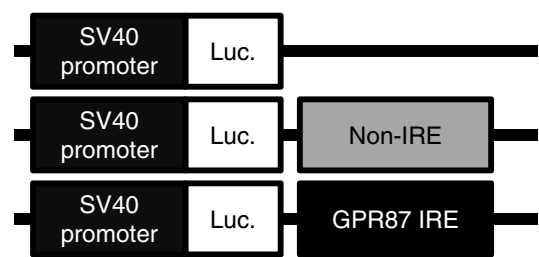

e

3C PCR

$$
\triangle \text { GPR87 IRE } \triangle \text { Non-IRE } \quad 3 \text { C PCR primer }
$$

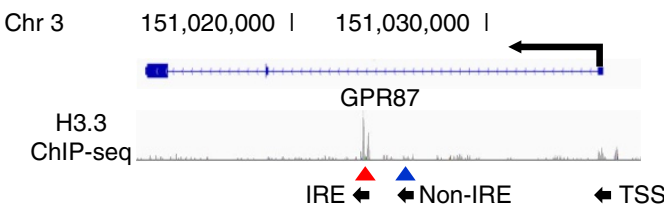

f

ChIP qPCR

$(-113 \sim+36$ bp from TSS $)$

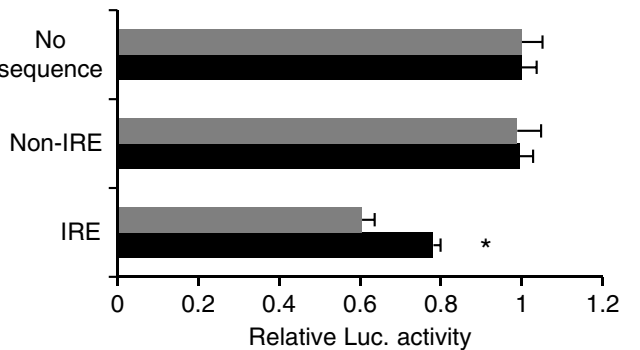

- Control

- H3F3A

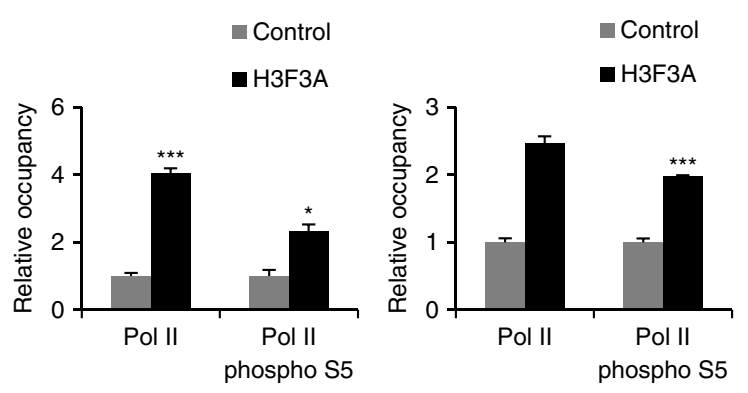

g

Invasion assay

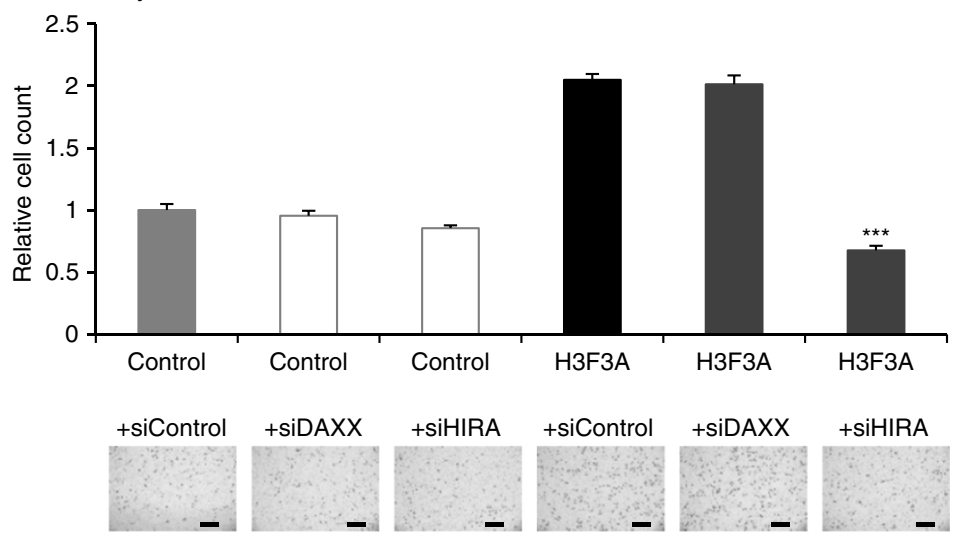

h

ChIP qPCR

(H3.3, GPR87 IRE)

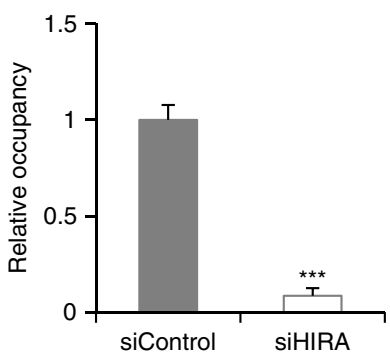

Figure 6 | Transcriptional regulation of GPR87 by H3F3A. (a) The element-directed reporter construct (GPR87 promoter). (b) The results of luciferase assays using the reporter (GPR87 promoter). (c) The element-directed reporter construct (SV40 promoter). (d) The results of luciferase assays using the reporter (SV40 promoter). (e) Validation of chromatin interaction changes between the GPR87 IRE and TSS due to H3F3A overexpression. (f) Validation of transcriptional machinery activation at the TSS by H3F3A overexpression. (g) Invasion assay with H3F3A overexpression after DAXX or HIRA knockdown. (h) ChIP-qPCR assay with H3F3A overexpression after HIRA knockdown (Flag ChIP at GPR87 IRE). Data are representative of three independent experiments. The error bars represent the s.e.m. ${ }^{\star} P<0.05,{ }^{\star \star} P<0.01,{ }^{\star \star \star} P<0.001, t$-test. Scale bar, $200 \mu \mathrm{m}$. 
a

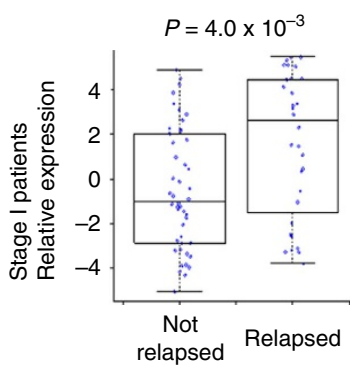

C

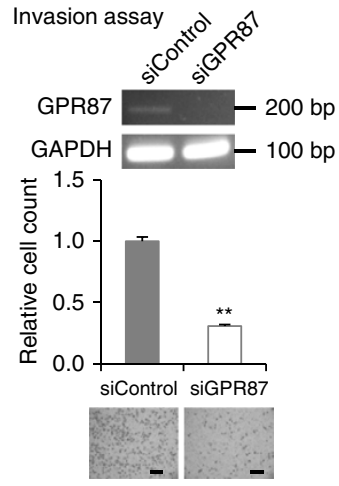

f

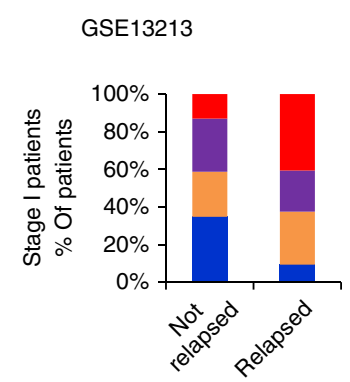

H3F3A: low, GPR87: low
GSE31210

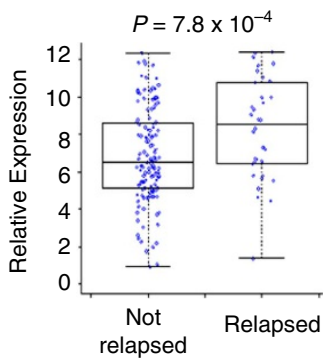

Proliferation Assay
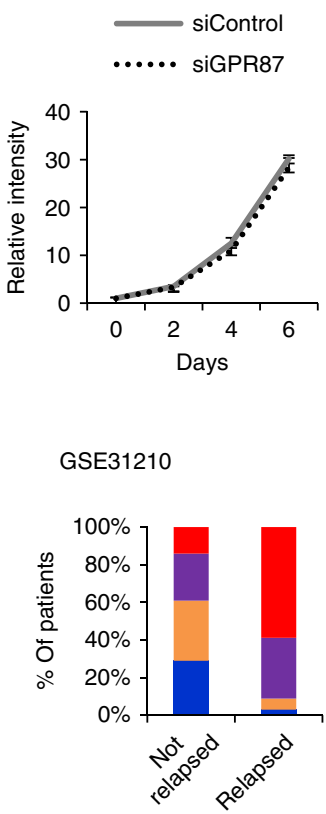

b GSE13213

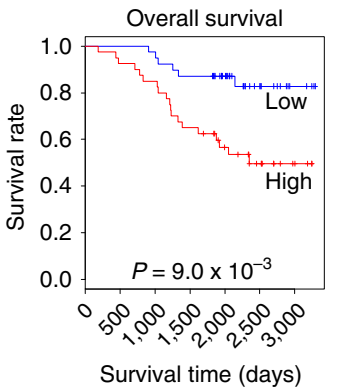

d
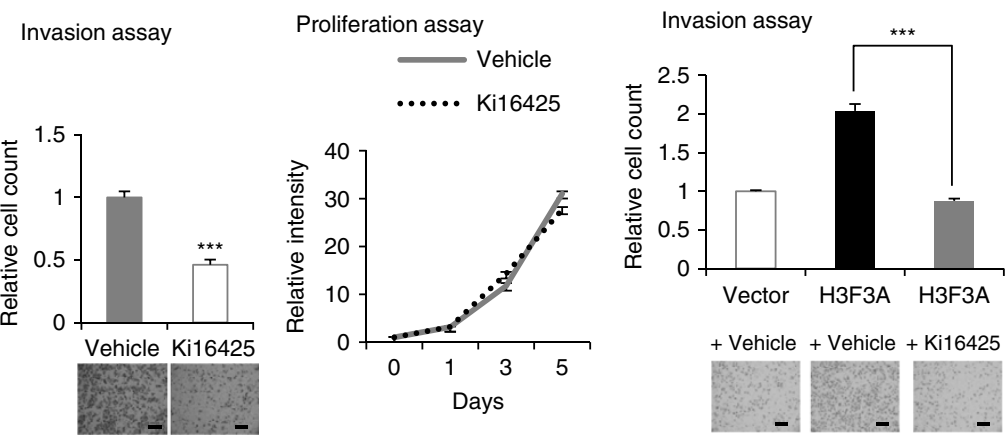

g GSE13213

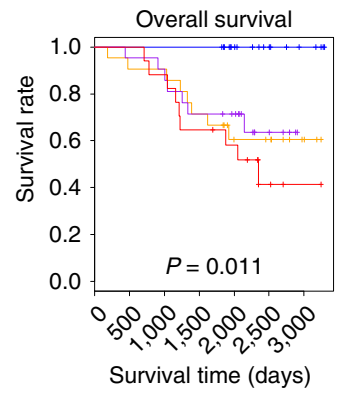

GSE31210
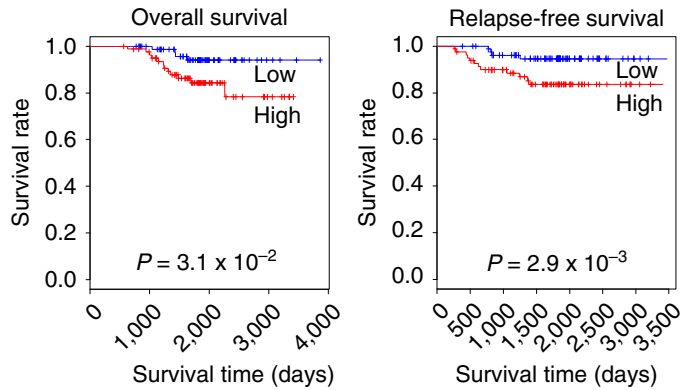

e

Survival time (days) 
cancer patients might be a viable strategy for post-operative adjuvant therapy.

Analysing the same data sets presented in Fig. 1, we examined the effect of the combination of H3F3A/GPR87 on relapse and patient prognosis. We divided the patients into four groups according to their H3F3A and GPR87 expression levels and then compared the portion of the four group patients between non-relapsed and relapsed patients (Fig. 7f). The relapsed patients contained a high proportion of patients in the high $H 3 F 3 A$ and high GPR87 expression group, and a low proportion in the low H3F3A and low GPR87 expression group in the both data sets. We also performed a survival analysis (Fig. $7 \mathrm{~g}$ and Supplementary Table 1). Surprisingly, all of the patients in the low $H 3 F 3 A$ and low GPR87 expression group were still alive. The patients in the high $H 3 F 3 A$ and high GPR87 expression group exhibited the worst prognoses. These results imply that the increase in GPR87 expression induced by $H 3 F 3 A$ overexpression plays a critical role in lung cancer progression. Thus, we suggest that the combination of $H 3 F 3 A / G P R 87$ expression is a novel prognosis marker for early-stage lung cancer.

\section{Discussion}

Histone variants can act as cancer drivers through the transcriptional activation or repression of cancer-related genes. For example, macroH2A suppresses melanoma progression by repressing the transcription of the cancer driver gene CDK8 (ref. 8). In this study, $H 3 F 3 A$ affects cancer progression by directly regulating the cancer driver gene GPR87, although in this case, it promotes cancer progression by acting as a transcriptional activator. Similar to $H 3 F 3 A$, the histone variant H2A.Z promotes cancer through transcriptional activation in various cancer types. The incorporation of H2A.Z into the promoter regions of oestrogen receptor (ERalpha) target genes (for example, TFF1) activates transcription in the presence of oestrogen in breast cancer cells ${ }^{9}$, and one isoform of H2A.Z mediates proliferation and drug sensitivity in melanoma by interacting with $B R D 2$ and $E 2 F$ target genes ${ }^{39}$. Regulatory regions containing both H3.3 and H2A.Z are known to be nucleosomefree regions that play distinct roles in transcriptional modulation ${ }^{16,22}$. According to our results, H3.3 is deposited at the GPR87 IRE, where it increases the occupancy of H2A.Z, modifies chromatin status and interactions, and directly activates GPR87 transcription. Therefore, this finding is a novel example of a histone variant acting as a transcriptional activator of cancer-promoting genes.

The well-known Lys27Met mutation in H3.3 acts as gain-offunction driver by inhibiting PRC2 activity in brain tumours ${ }^{17-20}$. Nevertheless, the Lys27Met mutation does not appear to be recurrent in lung cancer based on public cancer-related databases. Although the Lys27Met mutation indirectly upregulates the expression of target genes by inhibiting repressive $\mathrm{H} 3 \mathrm{~K} 27 \mathrm{me} 3$ modifications, H3.3 overexpression in lung cancer directly upregulates the expression of GPR87 by promoting the active histone modifications $\mathrm{H} 3 \mathrm{~K} 4 \mathrm{me} 1$ and $\mathrm{H} 3 \mathrm{~K} 9 \mathrm{ac}$, which are known to be important enhancer-associated chromatin modifications ${ }^{33}$. These results demonstrate that $\mathrm{H} 3.3$ overexpression increased the GPR87 IRE enhancer activity, which is a novel example of a direct mechanism enhancing the transcription of cancer-promoting genes.

In general, the function of $\mathrm{H} 3.3$ at promoter and intergenic enhancer regions has been relatively well characterized. At promoter regions, $\mathrm{H} 3.3$ primes and/or activates transcription by counteracting chromatin compaction, whereas at enhancers, it affects the transcriptional potential of target genes ${ }^{16}$. Although it is known that IREs have a large impact on transcriptional regulation by affecting histone modifications and chromatin remodelling, transcriptional regulation through $\mathrm{H} 3.3$ binding to the intronic regions of target genes has not been well studied ${ }^{26-28}$. Based on our integrated analyses, H3.3 deposition at intronic regions globally activates target gene expression. Moreover, globally, H3.3 binding regions showed characteristics of regulatory DNA elements and tended to contain active transcription histone marks. These results suggest that H3.3 is an important global regulator of transcription that can act through IREs.

GPR87 encodes a G-protein-coupled receptor and is a viable target molecule for cancer treatment and prevention ${ }^{36}$. GPR87 has been reported to promote growth, p53-dependent cell survival and the metastasis of human tumour cells in a variety of cancers ${ }^{31,40-42}$. However, upstream regulators of GPR87 have not been well characterized. We demonstrate that GPR87 expression is upregulated by $H 3 F 3 A$ overexpression and that $\mathrm{H} 3.3$ (expressed from $H 3 F 3 A$ ) is deposited at a specific intronic region of GPR87 in lung cancer. Moreover, by performing element-directed reporter and 3C-PCR assays, we describe in detail how H3.3 modifies the chromatin status of the GPR87 IRE to regulate the transcriptional activity of this gene. Our results reveal a novel upstream GPR87 regulator and elucidate an important transcriptional regulation mechanism in the context of lung cancer.

Two distinct genes, $H 3 F 3 A$ and $H 3 F 3 B$, both encode the identical protein $\mathrm{H} 3.3$, although they appear to be differentially regulated in cancer. For example, $H 3 F 3 A$ and $H 3 F 3 B$ show distinct recurrent mutations in cancer samples ${ }^{43}$, and they also possess different $5^{\prime}$ and $3^{\prime}$ UTR, exon-intron and promoter structures. Furthermore, $H 3 F 3 A$ and $H 3 F 3 B$ appear to be regulated by different transcriptional and post-transcriptional factors, such as microRNAs. We found that $H 3 F 3 B$ was associated with lung cancer progression in one data set but not in the other. We believe this result might have been due to differences between the two microarray platforms used for the GSE13213 and GSE31210 data sets. In particular, GSE13213 used a two-channel microarray platform (normal-tumour difference), whereas GSE31210 used a one-channel microarray platform (expression in tumours). Nevertheless, H3F3A showed a strong association with lung cancer progression in both data sets, indicating that $H 3 F 3 A$ is a robust prognostic marker.

Although the phenotypes were validated only for in vitro models and not for in vivo models, H3F3A and GPR87 both specifically influenced metastasis-related phenotypes but not growth-related phenotypes, suggesting that they mainly influence cancer progression and not tumorigenesis. Either gene alone is a good prognostic marker for early-stage lung cancer, although in combination, they are highly effective at identifying patients with good prognoses. Here, we elucidate the mechanism underlying the utility of H3F3A and GPR87 as prognostic markers by describing a novel process of transcriptional regulation. We evaluated the potential for these genes as prognostic markers and therapeutic targets using ectopic overexpression of $\mathrm{H} 3 \mathrm{~F} 3 \mathrm{~A}$ and an LPA antagonist, which inhibits GPR87. Our results indicate that using $H 3 F 3 A$ as a prognostic marker and GPR87 as a therapeutic target is a promising lung cancer treatment strategy. Considering that the combination of high $H 3 F 3 A$ and GPR87 expression is closely associated with lung cancer prognosis and that $H 3 F 3 A$ epigenetically regulates GPR87 transcription, combining LPA antagonists with epigenetic targeting of GPR87 transcription through H3.3 could be an effective strategy for cancer treatment, including post-operative therapy for lung cancer patients to decrease lung cancer-related deaths. Therefore, we suggest that GPR87 regulation by $H 3 F 3 A$ has translational potential for novel therapeutic strategies. 


\section{Methods}

Public data analysis. Public microarray data containing clinical information were downloaded from the NCBI GEO database (http://www.ncbi.nlm.nih.gov/geo/). The accession numbers of the collected data were GSE31210 and GSE13213. The data were globally normalized using the MAS5 method. Statistical tests were performed with the R programme, and survival analyses were performed with the R (https://www.r-project.org/) and APPEX (http://appex.kr/appex/) programs. Graphs and heatmaps were prepared using the Excel, R and MeV (http://www. tm4.org/mev.html) programs.

Public ChIP-Seq data were downloaded from the NCBI SRA (http://www. ncbi.nlm.nih.gov/sra/) and ENCODE databases (https://genome.ucsc.edu/ ENCODE/). The files from the NCBI SRA database were extracted to a fastq file using the SRA toolkit programme. The accession numbers of the NCBI SRA data were SRA043915, SRP008014 (GSE31794) and SRP019254 (GSE45023). The Bowtie2 (http://bowtie-bio.sourceforge.net/bowtie2/index.shtml) and MACS (http://liulab.dfci.harvard.edu/MACS/) programs were used for mapping to the GRCh37/hg19 reference genome and peak detection. The peaks were visualized with IGV (http://www.broadinstitute.org/software/igv/) and the UCSC genome browser (http://genome.ucsc.edu/)

Cell culture and transfection. A549 and NCI-H23 cells (ATCC) were maintained in complete RPMI 1,640 medium (Hyclone) at $37^{\circ} \mathrm{C}$ in a humidified $5 \% \mathrm{CO}_{2}$ incubator. The complete media was supplemented with $10 \%$ foetal bovine serum (Hyclone), $100 \mathrm{U} \mathrm{ml}^{-1}$ penicillin/streptomycin (WelGENE) and $2 \mathrm{mM}$ L-glutamine (Hyclone).

A Flag-tagged $H 3 F 3 A$ clone was constructed by PCR and inserted into the HRST-IRES-eGFP lentiviral vector. An empty vector was used as the control. The short hairpin RNA (shRNA) sequence for H3F3A was inserted into the pLL3.7 lentiviral vector. The target sequence of the shRNA was designed using the AsiDesigner programme (http://sysbio.kribb.re.kr:8080/AsiDesigner/). The shRNA sequences are listed in Supplementary Table 2. For viral particle generation, cloned DNAs, CMV delta 8.9 plasmid and VSV-G plasmid were transfected into 293T cells (ATCC) using Lipofectamin 2,000 (Invitrogen) in Opti-MEM media (Gibco). After $6 \mathrm{~h}$ of incubation, the media were changed to complete media. After $48 \mathrm{~h}$ of incubation, the supernatant containing the virus particles was harvested and concentrated. For viral infection, $1.5 \times 10^{5}$ lung cancer cells were added to each well of a 6-well plate and incubated overnight. The concentrated viral supernatant was added to the plated cells, and after overnight incubation, the media were changed to complete media. After $72 \mathrm{~h}$, the transfection was confirmed using a fluorescence microscope.

The siRNA target sequences were also designed using the AsiDesigner programme and the sequences of the transfected siRNAs are listed in Supplementary Table 2. For siRNA transfection, $1.5 \times 10^{5}$ lung cancer cells were plated in each well of a 6-well plate and incubated overnight. The siRNAs or non-targeting controls at a concentration of $50 \mathrm{nM}$ were transfected into lung cancer cells using Lipofectamin 2,000 in Opti-MEM media. After $4 \mathrm{~h}$ of incubation, the media were changed to complete media, and $48 \mathrm{~h}$ after transfection, the knockdown was confirmed by RT-PCR. Uncropped scans of the gel images are presented as Supplementary Fig. 12.

Reverse transcription PCR and qPCR. Total RNA was extracted using the RNeasy Mini Kit (QIAGEN) according to the manufacturer's instructions. Reverse transcription was performed with $1 \mu \mathrm{g}$ of total RNA as the template and M-MLV Reverse Transcriptase (Promega). RT-PCR assays were performed using the AmfiXpand PCR Master Mix (GenDEPOT). Quantitative real-time PCR (qPCR) reactions were performed in triplicate on a LightCycler 480 machine (Roche) using the LightCycler 480 SYBR Green I Master Mix (Roche). cDNA expression was normalized to the GAPDH or $\beta$-actin levels. At least three independent biological replicates were included in each PCR/qPCR. The primers used for the PCR/qPCR reactions were designed either manually or with the Primer3 programme (http://biotools.umassmed.edu/bioapps/primer3_www.cgi). All primer sequences are listed in Supplementary Table 3. Uncropped scans of the gel images are presented as Supplementary Fig. 12.

Immunoblot assay. Total protein samples were prepared in radioimmunoprecipitation assay buffer with protease inhibitor cocktail (Roche) and quantified using the Pierce BCA Protein Assay Kit (Thermo). Equal amounts of the samples were loaded onto 4-12\% Bis-Tris Protein Gels (Novex), electrophoresed, transferred to an Immobilon-P PVDF membrane (Millipore), and probed with the antibodies listed on Supplementary Table 4. HRP-conjugated anti-mouse or anti-rabbit IgG antibody (R\&D Systems) was used as the secondary antibody. The bands were detected with a WEST-ZOL plus Western Blot Detection System (iNtRON Biotechnology). Uncropped scans of the immunoblots are presented as Supplementary Fig. 12.

Invasion assay. Transwell chambers (Corning) were coated with Matrigel Basement Membrane Matrix (BD). Cells were suspended in serum-free media and seeded into the upper chamber at a density of 2.0 to $5.0 \times 10^{4}$ cells per well, and serum-containing media was placed into the lower chamber. After incubation for
$24 \mathrm{~h}$, the cells penetrating the pores were stained with Diff-Quik staining solution (Sysmex) and observed using a microscope.

Proliferation assay. Suspensions of 1.0 to $2.0 \times 10^{3}$ cells were seeded into 96 -well. After $0-144 \mathrm{~h}$ of incubation at $37^{\circ} \mathrm{C}$, a mixture of CyQUANT NF Cell Proliferation dye reagent and deliverer (Invitrogen) was added. After $4 \mathrm{~h}$ of incubation, the ratio of the fluorescence intensity of 530 to that at $485 \mathrm{~nm}$ was measured.

Microarray analysis. The Affymetrix Human ST2.0 microarray platform was used. Libraries were prepared from the total RNA samples $(2 \mu \mathrm{g})$ and hybridized, and the intensity was measured according to the manufacturer's instructions. The intensity values were globally normalized using the Robust Multi-array Average (RMA) method. Differential expression was tested using Student's $t$-test and fold change. A Gene Ontology analysis was performed using DAVID (http://david. abcc.ncifcrf.gov/). Graphs and heatmaps were prepared using the Excel, R and $\mathrm{MeV}$ programs.

Chromatin immunoprecipitation assays. ChIP was performed using the ChIP Assay Kit (Millipore) according to the manufacturer's instructions. For ChIP-PCR/ qPCR, 1.0 to $4.0 \times 10^{6}$ cells were cross-linked with $1 \%$ formaldehyde for $10 \mathrm{~min}$ at $25^{\circ} \mathrm{C}$. For H3.3 ChIP-Seq, $3.0 \times 10^{7}$ cells were prepared without cross-linking. The cells were resuspended and lysed for $10 \mathrm{~min}$ at $4{ }^{\circ} \mathrm{C}$ with the SDS Lysis Buffer provided in the kit. The lysate was aliquoted into a $1.5-\mathrm{ml}$ tube and sonicated using a Bioruptor sonicator (Diagenode). The fragment size was $\sim 150-500 \mathrm{bp}$. Samples were diluted 10 -fold ( 1.0 to $4.0 \times 10^{6}$ cells per tube) in the ChIP dilution buffer provided in the kit and pre-cleared with $75 \mu$ l of Salmon Sperm DNA/Protein A Agarose beads for $30 \mathrm{~min}$ at $4^{\circ} \mathrm{C}$. Primary antibody was added to the pre-cleared supernatant, and the mixture was incubated overnight at $4{ }^{\circ} \mathrm{C}$. The antibodies used for the ChIP assay are listed in Supplementary Table 4. Sixty microliters of Salmon Sperm DNA/Protein A Agarose beads were added to the samples, and the mixtures were incubated for $1 \mathrm{~h}$ at $4{ }^{\circ} \mathrm{C}$. The beads were subsequently washed using wash buffers (low-salt, high-salt, $\mathrm{LiCl}$ and TE buffers). After $15 \mathrm{~min}$ of incubation at $65^{\circ} \mathrm{C}$, the precipitated chromatin was eluted twice in $250 \mu$ l of elution buffer $\left(0.1 \mathrm{M} \mathrm{NaHCO}_{3}\right.$ and $1 \%$ SDS). Reverse cross-linking was performed for $4 \mathrm{~h}$ at $65^{\circ} \mathrm{C}$. The chromatin was then treated with RNase A for $1 \mathrm{~h}$ at $37^{\circ} \mathrm{C}$ and with Proteinase $\mathrm{K}$ for $1 \mathrm{~h}$ at $45^{\circ} \mathrm{C}$. The DNA was purified through phenol/chloroform extraction or with a QIAquick PCR Purification Kit (QIAGEN).

ChIP-PCR assays were performed using the AmfiXpand PCR Master Mix. ChIP-qPCR reactions were performed in triplicate on the LightCycler 480 machine using the LightCycler 480 SYBR Green I Master Mix. The data were normalized to the input levels. At least three independent biological replicates were included for each ChIP-PCR/qPCR. The primer sequences are listed in Supplementary Table 3. Uncropped scans of the gel images are presented as Supplementary Fig. 12.

ChIP-Seq and RNA-Seq. Sequencing libraries for ChIP-Seq were prepared from 1 ng of DNA using the ThruPLEX-FD Prep Kit (Rubicon Genomics) according to the manufacturer's instructions. Paired-end reads that were $76 \mathrm{bp}$ in length were sequenced using a NextSeq 500 Sequencing System (Illumina). The ChIP-Seq data were analyzes using the Bowtie2 and MACS programs for mapping to the GRCh37/hg19 reference genome and peak detection. The peaks were visualized with IGV and the UCSC genome browser.

Sequencing libraries for RNA-Seq were prepared from $2 \mu \mathrm{g}$ of total RNA using a TruSeq Stranded mRNA Sample Preparation kit (Illumina) according to the manufacturer's instructions. Paired-end reads that were $100 \mathrm{bp}$ in length were sequenced using the HiSeq 2,500 Sequencing System (Illumina). To analyse the RNA-Seq data, TopHat (http://ccb.jhu.edu/software/tophat/index.shtml) and Cufflinks (http://cufflinks.cbcb.umd.edu/manual.html) programs were used for short-read gapped alignment to the GRCh37/hg19 reference genome and ab initio assembly. The results were visualized with IGV and the UCSC genome browser.

Metagene analysis of introns and promoters. Exons, introns, promoters and intergenic regions were defined based on hg19 gene annotation. The top 3,000 peaks were selected based on the peak scores provided by the MACS programme. From the top 3,000 peaks, 871 high-stringency peaks, specifically those that displayed a ChIP/input ratio that was $>16\left(\log _{2}(\mathrm{ChIP} /\right.$ input ratio $\left.) \geq 4\right)$, were selected. Among the 871 high-stringency peaks, intronic, exonic and promoter peaks occupied 366 gene regions. Because the generally used transcription start/end sites are not good reference positions for calculating the binding profiles of intronic peaks, we used the summit positions or peak center positions provided by the MACS programme as reference positions. To calculate the binding profiles near peaks, we divided the region near the summit positions or peak center positions (for example, $-2 \mathrm{~kb}$ to $+2 \mathrm{~kb}$ from $\mathrm{H} 3.3$ summit) into 10- or 20-bp bins and calculated the reads per kilobase per million mapped reads (RPKM) value for each bins using the Pysam Python package or HOMER (hypergeometric optimization of motif enrichment). The average RPKM of each bin was plotted using the R Plot and Lowess functions $(f=0.3)$. A heatmap of the RPKM of each bin was drawn using the R and MEV programs. Boxplots for enrichment within the region were drawn using R software. 
Reporter assay. Cloned GPR87 intronic sequences were inserted into the pGL3 Luciferase Reporter Vector (downstream of the luciferase gene with the BamHI and SalI restriction enzyme sites). A luciferase assay is performed using the Dual-Luciferase Reporter assay system (Promega) according to the manufacturer's instructions. For transfection, $5.0 \times 10^{4}$ cells were plated into each well of a 24 -well plate. The luciferase reporters were co-transfected with the control plasmid encoding renilla luciferase into the plated cells. The luciferase activity was measured using a VICTOR Light luminometer (PerkinElmer). The transfection efficiencies were normalized using the renilla luciferase activity as the control.

Chromosome conformation capture (3C). First, $5.0 \times 10^{4}$ cells were cross-linked with $2 \%$ formaldehyde for $10 \mathrm{~min}$ at $25^{\circ} \mathrm{C}$. Five millilitres of NP-40 buffer $(10 \mathrm{mM}$ Tris- $\mathrm{HCl}, \mathrm{pH} 7.5,10 \mathrm{mM} \mathrm{NaCl}, 0.2 \% \mathrm{NP}-40$, protease inhibitor cocktail) were added to the cells, and the cells were incubated at $4{ }^{\circ} \mathrm{C}$ for $2 \mathrm{~h}$. The mixture was then centrifuged, and the pellet was resuspended in $0.5 \mathrm{ml}$ of $1.2 \mathrm{x}$ DpnII restriction enzyme buffer (New England Biolabs). Fifteen microliters of $10 \%$ SDS was added to the sample, and the mixture was incubated at $37^{\circ} \mathrm{C}$ for $1 \mathrm{~h}$. Forty microliters of $25 \%$ Triton-X100 was added, and the sample incubated at $37^{\circ} \mathrm{C}$ for $1 \mathrm{~h}$. The sample was digested with 400 units of DpnII restriction enzyme at $37^{\circ} \mathrm{C}$ for $\sim 18 \mathrm{~h}$. To deactivate DpnII, $80 \mu \mathrm{l}$ of $10 \%$ SDS was added to the sample, and the mixture was incubated at $65^{\circ} \mathrm{C}$ for $20 \mathrm{~min}$. Then, $6.125 \mathrm{ml}$ of $1.15 \times$ ligation buffer and $300 \mu \mathrm{l}$ of $25 \%$ Triton-X100 were added, and the sample was incubated at $37^{\circ} \mathrm{C}$ for $1 \mathrm{~h}$. The digested samples were ligated with 100 units of T4 DNA ligase (Promega) at $16^{\circ} \mathrm{C}$ for $4 \mathrm{~h}$ and then at $37^{\circ} \mathrm{C}$ for $30 \mathrm{~min}$. Reverse cross-linking was performed for overnight at $65^{\circ} \mathrm{C}$. The chromatin was then treated with RNase A for $1 \mathrm{~h}$ at $37^{\circ} \mathrm{C}$ and with Proteinase $\mathrm{K}$ for $1 \mathrm{~h}$ at $45^{\circ} \mathrm{C}$. The DNA was purified through phenol/chloroform extraction or with the QIAquick PCR Purification Kit.

3C-PCR assays were performed using the AmfiXpand PCR Master Mix. The data were normalized to 'internal' primers located in the GAPDH gene. At least three independent biological replicates were included for each 3C-PCR assay. The primer sequences are listed in Supplementary Table 3. Uncropped scans of the gel images are presented as Supplementary Fig. 12.

Data availabilty. Sequencing data have been deposited in the SRA with the accession number SRP078775. Micoarray data have been deposited in the GEO with the accession number GSE84555. All the relevant data are available from the corresponding author (Y.-J.K.).

\section{References}

1. Siegel, R., Naishadham, D. \& Jemal, A. Cancer statistics, 2013. CA Cancer I. Clin. 63, 11-30 (2013).

2. Hoffman, P. C., Mauer, A. M. \& Vokes, E. E. Lung cancer. Lancet 355, 479-485 (2000).

3. Nesbitt, J. C., Putnam, J. B. Jr., Walsh, G. L., Roth, J. A. \& Mountain, C. F. Survival in early-stage non-small cell lung cancer. Ann. Thorac. Surg. 60, 466-472 (1995)

4. Roselli, M. et al. Postsurgical chemotherapy in stage IB nonsmall cell lung cancer: long-term survival in a randomized study. Int. J. Cancer 119, 955-960 (2006).

5. Douillard, J. Y. et al. Adjuvant vinorelbine plus cisplatin versus observation in patients with completely resected stage IB-IIIA non-small-cell lung cancer (Adjuvant Navelbine International Trialist Association [ANITA]): a randomised controlled trial. Lancet Oncol. 7, 719-727 (2006).

6. Winton, T. et al. Vinorelbine plus cisplatin versus observation in resected non-small-cell lung cancer. N. Engl. J. Med. 352, 2589-2597 (2005).

7. Pignon, J. P. et al. Lung adjuvant cisplatin evaluation: a pooled analysis by the LACE Collaborative Group. J. Clin. Oncol. 26, 3552-3559 (2008).

8. Kapoor, A. et al. The histone variant macroH2A suppresses melanoma progression through regulation of CDK8. Nature 468, 1105-1109 (2010).

9. Gevry, N. et al. Histone H2A.Z is essential for estrogen receptor signaling. Genes Dev. 23, 1522-1533 (2009).

10. Dryhurst, D., McMullen, B., Fazli, L., Rennie, P. S. \& Ausio, J. Histone H2A.Z prepares the prostate specific antigen (PSA) gene for androgen receptor-mediated transcription and is upregulated in a model of prostate cancer progression. Cancer Lett. 315, 38-47 (2012).

11. Szenker, E., Ray-Gallet, D. \& Almouzni, G. The double face of the histone variant H3.3. Cell Res. 21, 421-434 (2011).

12. Chow, C. M. et al. Variant histone H3.3 marks promoters of transcriptionally active genes during mammalian cell division. EMBO Rep. 6, 354-360 (2005).

13. Mito, Y., Henikoff, J. G. \& Henikoff, S. Genome-scale profiling of histone H3.3 replacement patterns. Nat. Genet. 37, 1090-1097 (2005).

14. Wirbelauer, C., Bell, O. \& Schubeler, D. Variant histone H3.3 is deposited at sites of nucleosomal displacement throughout transcribed genes while active histone modifications show a promoter-proximal bias. Genes Dev. 19, 1761-1766 (2005).

15. Goldberg, A. D. et al. Distinct factors control histone variant H3.3 localization at specific genomic regions. Cell 140, 678-691 (2010).
16. Chen, P. et al. H3.3 actively marks enhancers and primes gene transcription via opening higher-ordered chromatin. Genes Dev. 27, 2109-2124 (2013).

17. Lewis, P. W. et al. Inhibition of PRC2 activity by a gain-of-function H3 mutation found in pediatric glioblastoma. Science 340, 857-861 (2013).

18. Schwartzentruber, J. et al. Driver mutations in histone $\mathrm{H} 3.3$ and chromatin remodelling genes in paediatric glioblastoma. Nature 482, 226-231 (2012).

19. $\mathrm{Wu}, \mathrm{G}$. et al. Somatic histone $\mathrm{H} 3$ alterations in pediatric diffuse intrinsic pontine gliomas and non-brainstem glioblastomas. Nat. Genet. 44, 251-253 (2012).

20. Chan, K. M. et al. The histone H3.3K27M mutation in pediatric glioma reprograms H3K27 methylation and gene expression. Genes Dev. 27, 985-990 (2013).

21. Graber, M. W., Schweinfest, C. W., Reed, C. E., Papas, T. S. \& Baron, P. L. Isolation of differentially expressed genes in carcinoma of the esophagus. Ann. Surg. Oncol. 3, 192-197 (1996).

22. Jin, C. et al. H3.3/H2A.Z double variant-containing nucleosomes mark 'nucleosome-free regions' of active promoters and other regulatory regions. Nat. Genet. 41, 941-945 (2009).

23. Ray-Gallet, D. et al. Dynamics of histone H3 deposition in vivo reveal a nucleosome gap-filling mechanism for $\mathrm{H} 3.3$ to maintain chromatin integrity. Mol. Cell 44, 928-941 (2011).

24. Adam, S., Polo, S. E. \& Almouzni, G. Transcription recovery after DNA damage requires chromatin priming by the $\mathrm{H} 3.3$ histone chaperone HIRA. Cell 155, 94-106 (2013).

25. Banaszynski, L. A. et al. Hira-dependent histone H3.3 deposition facilitates PRC2 recruitment at developmental loci in ES cells. Cell 155, 107-120 (2013).

26. Guo, Z., Boekhoudt, G. H. \& Boss, J. M. Role of the intronic enhancer in tumor necrosis factor-mediated induction of manganous superoxide dismutase. J. Biol. Chem. 278, 23570-23578 (2003).

27. Imamichi, Y. et al. Transcriptional regulation of human ferredoxin reductase through an intronic enhancer in steroidogenic cells. Biochim. Biophys. Acta 1839, 33-42 (2014)

28. Wang, H., Yan, C., Asangani, I., Allgayer, H. \& Boyd, D. D. Identification of an histone $\mathrm{H} 3$ acetylated/K4-methylated-bound intragenic enhancer regulatory for urokinase receptor expression. Oncogene 26, 2058-2070 (2007).

29. Tomida, S. et al. Relapse-related molecular signature in lung adenocarcinomas identifies patients with dismal prognosis. J. Clin. Oncol. 27, 2793-2799 (2009).

30. Okayama, H. et al. Identification of genes upregulated in ALK-positive and EGFR/KRAS/ALK-negative lung adenocarcinomas. Cancer Res. 72, 100-111 (2012).

31. Nii, K. et al. Overexpression of G protein-coupled receptor 87 correlates with poorer tumor differentiation and higher tumor proliferation in non-small-cell lung cancer. Mol. Clin. Oncol. 2, 539-544 (2014).

32. Pchelintsev, N. A. et al. Placing the HIRA histone chaperone complex in the chromatin landscape. Cell Rep. 3, 1012-1019 (2013).

33. Calo, E. \& Wysocka, J. Modification of enhancer chromatin: what, how, and why? Mol. Cell 49, 825-837 (2013).

34. Huang, C. et al. H3.3-H4 tetramer splitting events feature cell-type specific enhancers. PLoS Genet. 9, e1003558 (2013).

35. Kraushaar, D. C. et al. Genome-wide incorporation dynamics reveal distinct categories of turnover for the histone variant H3.3. Genome Biol. 14, R121 (2013).

36. Zhang, Y., Scoumanne, A. \& Chen, X. G protein-coupled receptor 87: a promising opportunity for cancer drug discovery. Mol. Cell. Pharmacol. 2, 111-116 (2010).

37. Tabata, K., Baba, K., Shiraishi, A., Ito, M. \& Fujita, N. The orphan GPCR GPR87 was deorphanized and shown to be a lysophosphatidic acid receptor. Biochem. Biophys. Res. Commun. 363, 861-866 (2007).

38. Ochiai, S., Furuta, D., Sugita, K., Taniura, H. \& Fujita, N. GPR87 mediates lysophosphatidic acid-induced colony dispersal in A431 cells. Eur. J. Pharmacol. 715, 15-20 (2013).

39. Vardabasso, C. et al. Histone variant H2A.Z.2 mediates proliferation and drug sensitivity of malignant melanoma. Mol. Cell 59, 75-88 (2015).

40. Glatt, S. et al. hGPR87 contributes to viability of human tumor cells. Int. J. Cancer 122, 2008-2016 (2008).

41. Zhang, Y., Qian, Y., Lu, W. \& Chen, X. The G protein-coupled receptor 87 is necessary for p53-dependent cell survival in response to genotoxic stress. Cancer Res. 69, 6049-6056 (2009).

42. Yan, M. et al. G protein-coupled receptor 87 (GPR87) promotes the growth and metastasis of $\mathrm{CD} 133(+)$ cancer stem-like cells in hepatocellular carcinoma. PLoS ONE 8, e61056 (2013).

43. Lindroth, A. M. \& Plass, C. Recurrent H3.3 alterations in childhood tumors. Nat. Genet. 45, 1413-1414 (2013).

\section{Acknowledgements}

This work was supported by grants from the National Cancer Center, Republic of Korea (NCC-1410300-1 and NCC-1210360-2), Basic Science Research Programme through the National Research Foundation of Korea (NRF) funded by the Ministry of Science, 
ICT \& Future Planning (2015R1C1A1A02036803) and the Korean Health Technology R\&D Project, Ministry of Health \& Welfare (HI13C2143)

\section{Author contributions}

J.K.C., Y.-J.K., S.-H.L. and I.-H.K. conceived this study. S.-M.P., E.-Y.C. and Y.-J.K. designed the experiments. S.-M.P., E.-Y.C. and S.K. performed the experiments. S.-M.P., E.-Y.C., M.B. and Y.-J.K. analysed data with input from J.B.P., H.Y. and S.-H.L.; S.-M.P., E.-Y.C., Y.-J.K. and S.-H.L. wrote the manuscript.

\section{Additional information}

Supplementary Information accompanies this paper at http://www.nature.com/ naturecommunications

Competing financial interests: The authors declare no competing financial interests.
Reprints and permission information is available online at http://npg.nature.com/ reprintsandpermissions/

How to cite this article: Park, S.-M. et al. Histone variant H3F3A promotes lung cancer cell migration through intronic regulation. Nat. Commun. 7, 12914 doi: $10.1038 /$ ncomms12914 (2016).

(c) (i) This work is licensed under a Creative Commons Attribution 4.0 International License. The images or other third party material in this article are included in the article's Creative Commons license, unless indicated otherwise in the credit line; if the material is not included under the Creative Commons license, users will need to obtain permission from the license holder to reproduce the material To view a copy of this license, visit http://creativecommons.org/licenses/by/4.0/

(C) The Author(s) 2016 